University of Warwick institutional repository: http://go.warwick.ac.uk/wrap This paper is made available online in accordance with publisher policies. Please scroll down to view the document itself. Please refer to the repository record for this item and our policy information available from the repository home page for further information.

To see the final version of this paper please visit the publisher's website. Access to the published version may require a subscription.

Author(s): NATALIA L. KOMAROVA and ALAN C. NEWELL

Article Title: Nonlinear dynamics of sand banks and sand waves

Year of publication: 2000

Link to published version:

http://dx.doi.org/10.1017/S0022112000008855

Publisher statement: None 


\title{
Nonlinear dynamics of sand banks and sand waves
}

\author{
By NATALIA L. KOMAROVA ${ }^{1,2}$ \\ AND ALAN C. NEWEL L ${ }^{1,3}$ \\ ${ }^{1}$ Department of Mathematics, University of Warwick, Coventry, CV4 7AL, UK \\ ${ }^{2}$ Institute for Advanced Study, School of Mathematics, Einstein Drive, Princeton, NJ 08540, USA \\ ${ }^{3}$ Department of Mathematics, University of Arizona, Tucson, AZ 85721, USA \\ (Received 13 January 1999 and in revised form 28 February 2000)
}

Sand banks and sand waves are two types of sand structures that are commonly observed on an off-shore sea bed. We describe the formation of these features using the equations of the fluid motion coupled with the mass conservation law for the sediment transport. The bottom features are a result of an instability due to tidebottom interactions. There are at least two mechanisms responsible for the growth of sand banks and sand waves. One is linear instability, and the other is nonlinear coupling between long sand banks and short sand waves. One novel feature of this work is the suggestion that the latter is more important for the generation of sand banks. We derive nonlinear amplitude equations governing the coupled dynamics of sand waves and sand banks. Based on these equations, we estimate characteristic features for sand banks and find that the estimates are consistent with measurements.

\section{Introduction}

\subsection{General}

Water motion over a sandy bed often leads to formation of various regular bottom structures. Examples of such features are sand bars in straight channels (see Schielen, Doelman \& De Swart 1993; Komarova \& Newell 1995); small sand ripples under sea waves (Blondeaux 1990); and sand waves and sand banks in tidal seas (Hulscher 1996; Hulscher, De Swart \& De Vriend 1993). These features occur on very different scales and have different characteristics. Table 1 gives definitions of some sand patterns. It includes their typical sizes and the kind of water flows which are thought to be responsible for the bed feature generation. It is interesting that some of the patterns listed in table 1 can coexist in nature. For instance, sand waves are often observed on top of tidal banks (see Huntley et al. 1993; Stride 1982 and figure 1). The coexistence of alternate bars and a mean flow component, the last two patterns of table 1, has been demonstrated. Ripples and megaripples are often seen together near the shore line, where sea waves ebb and flow. In each of these situations, the two coexisting features have vastly different wavelengths. These observations lead us to ask whether long and short waves are generated independently or whether they are dynamically coupled so that the appearance of short-scale waves requires the presence of longer-scale features.

To describe the formation of sand patterns under water, one has to couple the hydrodynamic equations of motion with an equation describing the behaviour of sand. The equations of motion for water derive from the Navier-Stokes equation by means of appropriate approximations (shallow water, deep water) and relevant 


\begin{tabular}{llll}
\hline \multicolumn{1}{c}{ Name } & \multicolumn{1}{c}{ Flow } & \multicolumn{1}{c}{ Size } & \multicolumn{1}{c}{ References } \\
Ripples & Sea waves & $6-12 \mathrm{~cm}$ & Blondeaux (1990) \\
Megaripples & Sea waves & $1-5 \mathrm{~m}$ & Knaapen (1999), Larcombe \& Jago (1996) \\
Sand waves & Tides & $200-800 \mathrm{~m}$ & Hulscher (1996), Huntley et al. (1993) \\
Tidal banks & Tides & $2-10 \mathrm{~km}$ & Hulscher (1996), Huntley et al. (1993) \\
Alternate bars & Unidirectional flow & $5-10 \mathrm{~m}$ & Schielen et al. (1993) \\
'Mean flow' & Unidirectional flow & $50-100 \mathrm{~m}$ & Komarova \& Newell (1995) \\
& \multicolumn{2}{r}{ TABLE 1. Definitions of sand patterns. } \\
\hline
\end{tabular}

boundary conditions. To describe the bed profile, a mass conservation law based on an empirical formula for the sediment flux is usually used. Despite a variety of formulations, one common feature can be found in all such systems, namely the existence of a soft mode arising from a common symmetry. The soft mode in the sand ripple/megaripple and sand wave/sand bank systems is the vertical translation of the sea bed. It is represented by a deformation of the sea bed which varies significantly only over distances long compared with the wavelength excited directly by instability. Through nonlinearity, this mode is driven by slow variations in the intensity of the fields of the unstable sand ripples or sand waves. This result is very general and is not affected by the fact that the basic instability mechanism might differ from system to system.

In this paper, we study the nonlinear generation of sand banks by sand waves. Whereas linear stability theory (see Hulscher 1996) does reasonably well in predicting the onset and presence of sand waves, it has been less successful in explaining the simultaneous appearance of sand banks, which are up to ten times larger. The available theories (see Hulscher et al. 1993; De Vriend 1990; and Dyer \& Huntley 1999 for reviews on sand bank generation) predict a growth-rate time scale of about 200 years. According to these theories, sand banks and sand waves are created independently by different physical mechanisms. On the other hand, it is observed that sand banks and sand waves occur together (in fact, in Stride 1982 it is reported that tidal banks never occur without sand waves). Also, the equation for the deformation $h$ of the sand bed contains spatial derivatives of quadratic products of the bottom stress, which is a functional of $h$ determined by solving the hydrodynamical equations. When averaged over the sand-wave scale, this equation gives rise to terms analogous to the Reynolds stress in hydrodynamics and the ponderomotive force in plasma physics. These terms are proportional to the curvature of the sand wave intensity and drive a long-wave deformation of the sand bed. We prove that the time it takes the sand bank deformation to grow to a height of several metres is about 10 years, which is 20 times faster than the rate suggested by linear theories.

\subsection{The main ideas}

We take a two-dimensional model with $x$ denoting the horizontal coordinate parallel to the tide and $z$ the vertical coordinate. The sand bed is denoted by $z=h(x, t)$. The time dependence of $h(x, t)$ is of the order of years or about $10^{4}$ times the tidal period $2 \pi / \Omega$. The spatial structure of interest is a superposition of a sand wave packet with carrier number $k_{c}$ (corresponding to a wavelength of approximately 200-800 m) chosen by linear stability considerations, and a large-scale deformation; namely,

$$
h(x, t)=A(x, t) \mathrm{e}^{\mathrm{i} k_{c} x}+\text { c.c. }+B(x, t),
$$




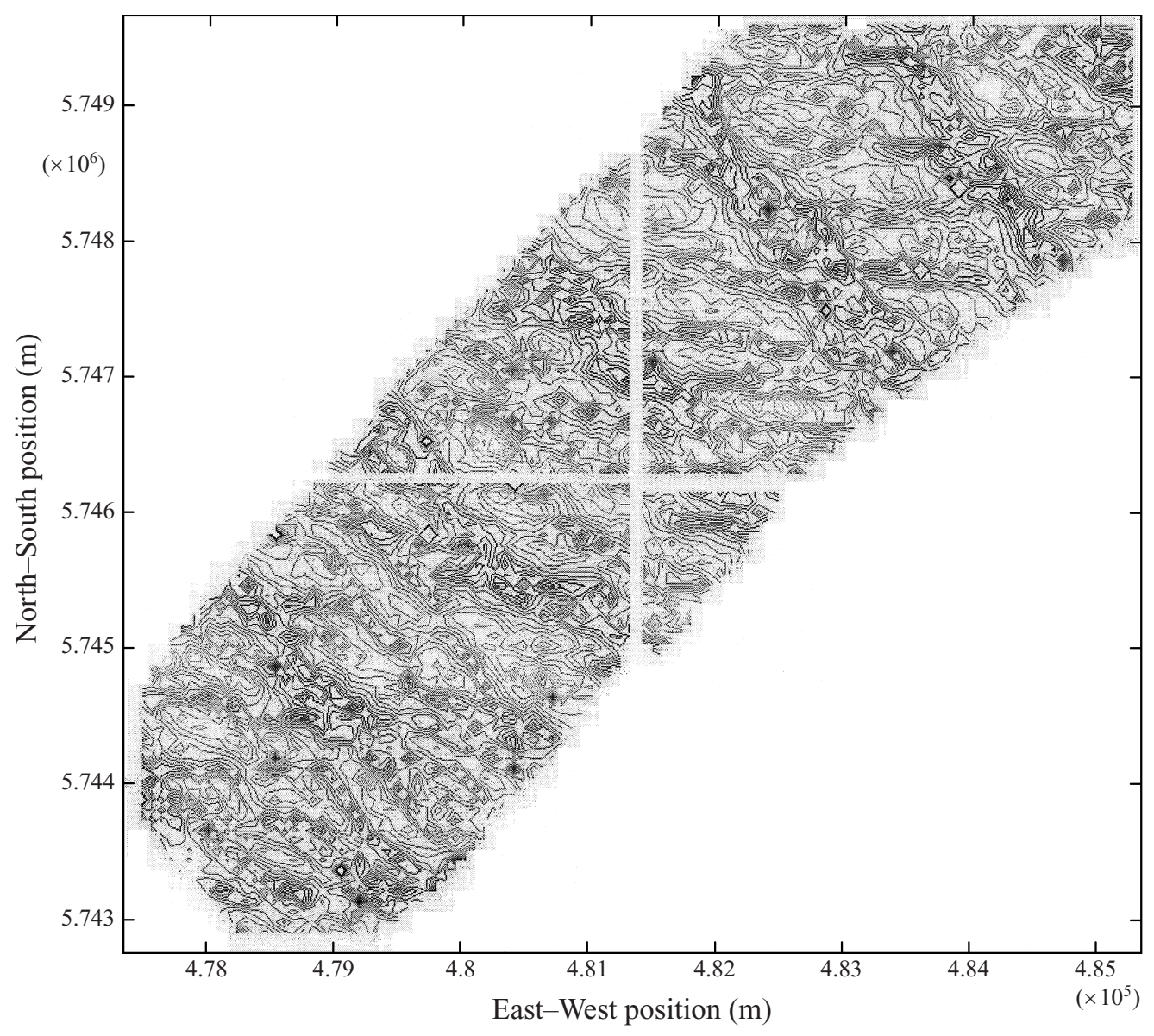

FIGURE 1. A contour plot of a part of the North Sea bed, based on measurements performed in 1990. Lighter contour lines correspond to deeper water. The direction of the tide is approximately North-South. Courtesy of Michiel A. F. Knaapen.

where c.c. stands for complex conjugate. Deformations in the sand bed are driven by a horizontal stress applied to the bed by circulating eddies. The eddies are in turn induced by averaging the effects of the interaction between the tidal motion and the bed profile over a tidal period. Consider figure 2. In its left to right cycle, the tidal motion encounters a favourable pressure gradient from trough $T_{1}$ to crest $C$ and an adverse one from $C$ to $T_{2}$. On the right to left cycle, the favourable pressure gradient is from $T_{2}$ to $C$ and the adverse from $C$ to $T_{1}$. This asymmetry means that, when averaged over a full cycle, the pressure gradient acts to speed up the flow from trough to crest near the sand bed. The steady-state circulations shown in figure 2 are the result of this action (see also Komarova \& Hulscher 2000 for a detailed study of this process). If these circulations are sufficiently strong to overcome the stabilizing influence of gravity (sand grains will tend to roll downhill from the crests to the troughs), the deformation shown in figure 2 can be amplified until saturated at some amplitude level by negative nonlinear feedback. This action is described by the sediment transport equation,

$$
\partial_{t} h=-\partial_{x}\langle q\rangle, \quad q \propto|\tau|^{b}\left(\tau-\lambda \partial_{x} h\right),
$$




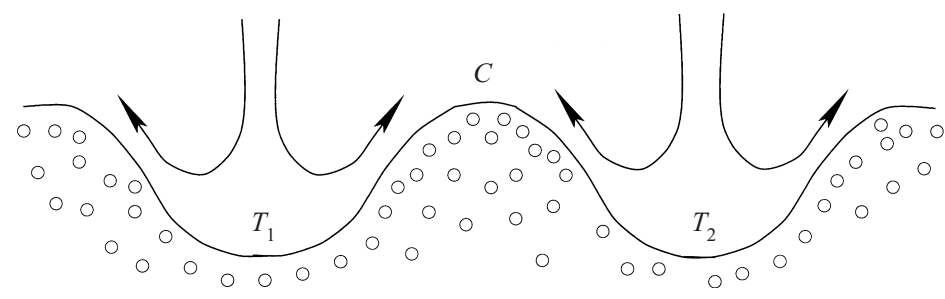

Figure 2. Basic instability mechanism. The arrows indicate the direction of the steady near-bed flow caused by the bed deformation. The time-periodic tidal flow is not shown.

where the volumetric flux $q$ is averaged $\langle\cdot\rangle$ over a tidal period, and $\tau$ is the volumetric stress tangential to the bed applied by the circulating cells. The parameter $\lambda$ measures the ratio of gravity to tidal strength. When it falls below a critical value $\lambda_{c}$, instability is triggered and sand waves appear. The parameters $\lambda$ and $b$ will be discussed later. Equation (1.2) is the empirical law of bed load transport (see e.g. Bailard 1981; Bailard \& Inman 1981; Falques, Montoto \& Iranzo 1996; Schielen et al. 1993; Hulscher 1996) which takes into account the traction (creeping) and saltation (skipping) of sand particles but not their transport by suspension. Other authors (Fredsoe 1974; Soulsby 1997; Schuttelaars \& De Swart 1996) have included the effects of suspension, but here the Froude number $U / \sqrt{g H}(U$ is the tidal velocity amplitude, $g$ is acceleration due to gravity and $H$ is the unperturbed depth) is too small, which indicates that there is much less suspended sediment in water (see Fredsoe \& Engelund 1975). Also, Hjulstrom's diagram (see e.g. Leeder 1982) suggests that for realistic values of the near-bed velocities and particle sizes, the prevailing mechanisms of sediment transport are the bed-load mechanisms.

The role of hydrodynamics is to provide the algorithm by which we compute the tangential shear stress as functional of the deformation $h, \tau=\tau[h]$. We begin with a tidal flow, $\boldsymbol{u}=\left(u(x, z, t)=u_{0}(z, t), w(x, z, t)=0\right)$ on $0 \leqslant z \leqslant H$. We perturb the bottom with a small-amplitude zero mean deformation $h$ and adiabatically (because changes in $h$ are so slow) calculate the corresponding deformations in the flow velocity $\boldsymbol{u}$ and pressure $p$ fields. Although complicated, there is nothing singular about this calculation. We end up with $\tau=\tau[h]$, namely with an expression for the shear stress which depends on $h$ and its $x$-derivatives. We then solve equation (1.2) for $h$ as a perturbation series in amplitude with the first terms given by expression (1.1). The variations of $A$ and $B$ with time are chosen to keep the asymptotic expansion uniformly valid in time. $A$, the envelope of the excited wave packet (whose width $\Delta k$ is proportional to the amplitude), and $B$ are both slowly varying in $x$, namely $\partial_{x} A / A$ and $\partial_{x} B / B$ are of order $A$. The perturbation series for $\partial_{t} A$ and $\partial_{t} B$ are Taylor series in $A, B$ and the slow derivative $\partial_{x}$. Simple arguments based on symmetry considerations tell us which terms can and cannot be present. These are familiar in the derivation of the class of 'amplitude', 'Newell-Whitehead-Segel', 'complex Ginzburg-Landau' equations which are part of a vast literature (Newell, Passot \& Lega 1993; Cross \& Hohenberg 1993) on the post-stability behaviour of pattern forming systems. For example, in the equation for $\partial_{t} B$, we seek those terms arising from the time-averaged flux $\langle q\rangle$, which have no fast $\left(\propto \mathrm{e}^{\mathrm{i} k_{c} x}\right.$ and higher harmonics) $x$-dependence. The first candidate would be $|A|^{2}$, but this is ruled out by symmetry considerations as a constant-intensity sand wave packet cannot produce a right or left net flux of sediment. The next available candidate is $\partial_{x}|A|^{2}$ which is allowed because, from a modification of the previous argument, sand waves with varying intensity can 


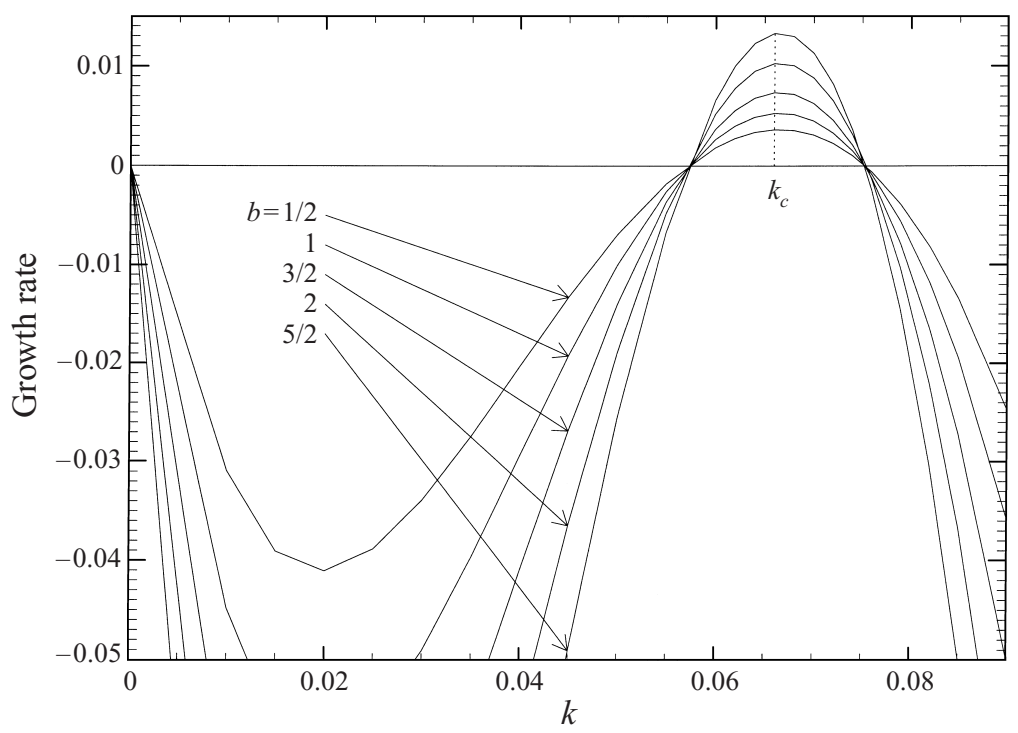

FIGURE 3. The growth rate curves for parameter set (i) of figure 7 and different values of $b$. The local minimum of the curve with $b=5 / 2$ is $\Gamma \approx-0.15$ (it corresponds to $k \approx 0.02$ ).

indeed drive the circulation shown in figure 2. Dependence of $\langle q\rangle$ on $B$ is ruled out because a constant change of sand bed level cannot produce a net flux. Therefore, the first term in $\langle q\rangle$ that depends on $B$ is $\partial_{x} B$ (and therefore, the first term in $\partial_{t} B$ is $\partial_{x}^{2} B$ ). The sign is important, and we will remark on this later in more detail. In our analysis it is positive, which means that long waves are damped. Therefore, the growth rate $\Gamma(k)$ as function of $k$ for the linear stability of the state $k=0, \boldsymbol{u}=\left(u_{0}(z, t), 0\right)$ will look like that shown in figure 3 . In this case, a time-independent state can be reached when $B$ is of the same order as $|A|^{2}$. The leading-order terms in the equation for $\partial_{t} A$, obtained as the $x$-derivative of the coefficient of $\mathrm{e}^{\mathrm{i} k_{c} x}$ in $\langle q\rangle$, are $\partial \Gamma / \partial \lambda_{c}\left(\lambda-\lambda_{c}\right) A, \partial_{x}^{2} A$ (with a positive coefficient reflecting the curvature of $\Gamma(k)$ at $k=k_{c}$ ), $|A|^{2} A$ and $B A$. Since the instability is not a travelling wave, the left-right symmetry in not broken, so no (group velocity) terms proportional to $\partial_{x} A$ appear.

The canonical equations we obtain are

$$
\begin{aligned}
& A_{T_{m}}=\sigma\left(\lambda_{c}-\lambda\right) / \lambda_{c} A+\gamma A_{x x}+c|A|^{2} A+\eta A B, \\
& B_{T_{m}}=\beta B_{x x}+\xi|A|_{x x}^{2},
\end{aligned}
$$

and it is the goal of the following analysis to determine the coefficients. The main point to be made, however, is that, independent of the details of the model, very long-wave deformations $B$ are driven by the gradients of sand wave intensities in the time-averaged flux $\langle q\rangle$.

The outline of the paper is as follows. In $\S 2$, we present the hydrodynamical model and discuss the eddy viscosity parameterization and approximations that we use. In $\S 3$, we give the basic solution corresponding to the tide over a flat bottom and perform the linear analysis of the perturbed system. We find the solutions corresponding to the most excited mode and the zero mode, and discuss the growth rate curve. Section 4 contains the nonlinear analysis of the system. We derive a set of coupled evolution equations for the two functions of interest: the envelope of sand waves and the amplitude of sand banks. The coefficients in the equations are defined 
through physical parameters of the system. Based on these equations we can predict the characteristic time for sand bank formation as well as the heights of sand banks and sand waves, which is done in $\S 5$. We also discuss various scenarios and conclude that predictions of our nonlinear model coincide reasonably well with observations. Conclusions are presented in $\S 6$.

\section{Details of the model}

Because we are dealing with a strongly turbulent motion, because the sediment transport equation is empirical and because the coupling between the two involves the prescription of the stress at the top of the molecular boundary layer, we have to recognize that the model is largely phenomenological. This means that while we choose the model to be consistent with the equations of fluid motion, it will also contain certain parameters connected with the characterization of viscosity, the sediment transport and the relation between bottom tangential stress and fluid velocity. Their values are chosen to belong to a range which gives results consistent with observations of sediment flux and sand wave fields in fairly shallow seas. But they cannot be precisely determined. Therefore, the model should be robust in that its predictions should not change qualitatively as the parameters in the phenomenological equations are varied throughout their likely ranges. In this way, improvements in the model prescription (a better characterization of viscosity or sediment flux) will not qualitatively change the conclusions. In particular, we want to make sure that the main prediction of this paper, the rate of generation of sand banks, is relatively insensitive to model details. We now discuss three of the main components, i.e. a characterization of the turbulent eddy viscosity which properly reflects changes due to bed deformations, the hydrodynamics and the sediment transport.

\subsection{Eddy viscosity}

It is important to recognize that even when the sand bed is flat, the tidal flow is highly turbulent. The Reynolds number based on molecular viscosity is enormous (tidal velocity, $U \approx 1 \mathrm{~m} \mathrm{~s}^{-1}$, the depth $H$ is of the order $50 \mathrm{~m}$ and molecular viscosity $v_{\text {mol }} \approx 2 \times 10^{-6} \mathrm{~m}^{2} \mathrm{~s}^{-1}$, so $R e \sim 10^{7}$ ), and the tidal solution is highly inflectional. Therefore, the turbulence Reynolds stresses greatly increase momentum transport. This feature is captured by using a turbulent eddy viscosity $v_{t} \sim 10^{-3}-10^{-2} \mathrm{~m}^{2} \mathrm{~s}^{-1}$ (see e.g. Pedlosky 1987). The quantity $v_{t}$ characterizes the typical distance, $\delta=\sqrt{2 v_{t} / \Omega}$, over which the (averaged) tidal flow changes. We will call $\delta$ the turbulent boundary layer thickness. In shallow tidal seas, it has the same order of magnitude as the depth $H$.

There are various ways to incorporate this idea in the hydrodynamical model. The simplest phenomenological model assumes that $v_{t}$ is constant (see e.g. Hulscher 1996; Maas \& van Haren 1987). Indeed, for simple unidirectional or harmonic (tidal) flows over a flat bed, the assumption that $\delta=$ const is not an unreasonable one. However, the 'amount of turbulence' in the water flow can acquire a spatial dependence if the bed is non-uniform and/or if the flow changes in the horizontal direction. Indeed, an analysis based on a constant-viscosity model gives a growth rate curve $\Gamma(k)$ which is positive over a range $0<k<k_{0}$, and at onset very long deformations are preferentially amplified. This is not consistent with observations (e.g. Huntley et al. 1993) in which sand waves belonging to a range of finite wavelengths seem to be preferred. The most obvious deficiency is the uniform characterization of the eddy viscosity. The viscosity model which we choose in this work reflects the fact that the 
strength of the turbulent eddies in the turbulent boundary layer has small corrections due to (i) deformations of the bed, and (ii) periodic time dependence of the tidal motion. Effectively this means that $v_{t}(x, t)=v_{0}(1+v)$ with

$$
v(x, t)=h \alpha_{1}(k)+h_{x} \alpha_{2}(k) u(h, t),
$$

where $\alpha_{1}(k)$ and $\alpha_{2}(k)$ are some functions of $k$ whose shape we will discuss. This choice is consistent with symmetries of the system (it is invariant under the transformation $x \rightarrow-x$ and $u \rightarrow-u$ ), and also it has a physical interpretation. The constant $v_{0}$ corresponds to the eddy viscosity associated with an unperturbed tidal motion over a flat bed. The first term on the right-hand side of equation (2.1) is time independent and reflects the part of the viscosity changes induced by the bed topography. The second term results from the interaction of the flow with the bed profile and therefore its time dependence is the same as the time dependence of the flow itself. It turns out that the coefficient $\alpha_{1}(k)$ plays a more important role in the long-wave behaviour of the system (so that effectively we are introducing a depth-dependent turbulent viscosity). Namely, if $\alpha_{1}(k)$ is finite and negative for small values of $k$, then this provides a mechanism for suppression of the ultra-long waves in the system. Physically $\alpha_{1}<0$ means that the eddy viscosity is slightly smaller over the crests of the sand waves than it is in the troughs. Note that if $h=\hat{h} \cos k x$, then the second term in expression (2.1) is vanishingly small for small values of $k$ and therefore does not play a significant role in the damping of long waves.

Viscosity Model I. In order to relate coefficients $\alpha_{1,2}$ to the physical parameters of the system, we constructed a simple example of a viscosity parameterization which, when linearized about the perturbed tidal state, coincides with representation (2.1). In this example, the eddy viscosity is a functional of the flow velocity. Our choice is purely phenomenological but it is related to the mixing-length concept first introduced by Prandtl (1932) (see also Soulsby 1990; Engelund 1970). As we show in $\S 2.2$, it is approximately consistent with the notion of a constant slip and a bottom shear stress proportional to $|u| u$. We take

$$
v_{t}=c_{1} H u_{n b},
$$

where $c_{1}$ is a constant, and the near-bed velocity is $u_{n b}=|u(z=d)|_{\text {trunc }}$, i.e. the absolute value of the velocity measured at some level $z=d$ close to the bed. Note that as long as $d$ is much smaller than the local depth, its concrete values do not change the results qualitatively (taking different values of $d$ is similar to redefining the constant $c_{1}$ ). In the present paper we took $d=0$. The truncation procedure used in expression (2.2) can be explained as follows. The turbulent boundary layer time evolution is completely determined by the flow. In the case of periodic flows, it means that the corresponding expression for the eddy viscosity cannot contain time harmonics higher than the ones present in the flow. Therefore, we truncate the expression $|u(z=d)|$ and only keep as many harmonics as are present in the function $u(z=d)$ itself. The constant $c_{1}$ is taken to give reasonable values for the turbulent viscosity (in particular, they coincide with the values used in Hulscher 1996 and Pedlosky 1987). In the text below we will refer to expression (2.2) as Model I. In the next section we give the expressions for coefficients $\alpha_{1}$ and $\alpha_{2}$ in this case. It turns out that $\alpha_{1}<0$.

Viscosity Model II. Another example of formula (2.1) is obtained by taking both coefficients $\alpha_{1,2}$ to be external constants of the system. The choice is not unreasonable because, for Model I, $\alpha_{1}$ is almost constant and we find from later calculations that the terms proportional to $\alpha_{2}$ contribute little to the outcome. It is useful, however, to consider this model (called Model II) in order to show that our results do not depend 
strongly on magnitudes in the choice of viscosity parameterization. They do, as noted above, depend on the sign of $\alpha_{1}$.

We would like to emphasize that the viscosity models used here can be refined in a number of ways. For instance, a vertical dependence of $v_{t}$ can be included (see e.g. Tennekes \& Lumley 1972; Soulsby 1990). However, since the exact $z$-dependence of the viscosity is unknown, such a choice is also phenomenological and in any event will tend to a characterization akin to (2.1). The important thing, as we have stressed, is to make sure that the main predictions are relatively insensitive to model details.

\subsection{Hydrodynamic equations}

The hydrodynamics of the problem are described by Navier-Stokes equations for the velocity field $\boldsymbol{u}=\left(\partial_{z} \Psi,-\partial_{x} \Psi\right)$ with the viscosity term containing $v_{t}$ and boundary conditions at the surface and the bed. The scales are given by the natural parameters of the system: $U$ (the tidal velocity amplitude), $\Omega$ (the tidal frequency) and $\delta$ (the turbulent boundary layer thickness, $\delta=\sqrt{2 v_{0} / \Omega}$ ). The typical horizontal length relevant for sand waves is much larger than the water depth, and therefore all horizontal dependences are much slower than the vertical ones. This gives us a chance to simplify the equations by neglecting the second $x$-derivatives in comparison with the second $z$-derivatives (shallow water approximation). At the surface, we use the so-called rigid lid approximation (see e.g. Bryan 1969; Phillips 1977; Pinardi, Rosati $\&$ Pacanowski 1995) of zero tangential stress $\left(\Psi_{z z}=0\right)$ and zero normal velocity $\left(\Psi_{x}=0\right)$. This is a matter of convenience rather than necessity. The first boundary condition at the sand bed $z=h(x)$ is the kinematic condition that a fluid particle on the sand bed remains there, $d_{t}(z-h(x, t))=-\Psi_{x}-\Psi_{z} h_{x}=0$ (here we neglect the time dependence of $h$ ). The second boundary condition specifies the tangential stress at the sand bed. In the context of a $z$-independent turbulent viscosity model, we have to recognize that the tangential bed stress, $v_{t} \partial_{z} u$, is not zero but (see e.g. Parker 1976; Maas \& van Haren 1987) given by a quadratic function of the horizontal velocity. The relevant dimensionless boundary condition is then $\Psi_{z z}=S \Psi_{z}$, where the resistance parameter, $S$, is introduced so that $S \neq \infty$ means that there is a finite slip in the system (see Engelund 1970). The same bottom boundary condition was used in Maas \& van Haren (1987) where it was derived by linearization of

$$
\tau=v_{t} \partial_{z} u=C_{d}|u| u
$$

where $C_{d}$ is the drag coefficient. The boundary condition we use is phenomenological. It enables us to circumvent a detailed description of the flow profile within the Stokes boundary layer (which is about $10 \mathrm{~cm}$ in our case), see also Bowden (1983). In the present model, $v_{t}=c_{1} u_{n b}$ and by linearizing the right-hand side of (2.3) about $u_{n b}$, we can estimate the resistance parameter, $S=\delta C_{d} /\left(H c_{1}\right)$.

\subsection{Sediment transport}

The bottom profile is described according to a mass conservation law for sand, which involves an empirical formula for the sediment flux, equation (1.2). In this equation, only two main forces are taken into account which act on bed sediment grains. The first term shows the scraping effect of the drag force, and the second one represents the gravity component along the bed profile. In order to give some estimates and define the morphological time scale, $\Delta T$, we will write down the expression for sediment flux in dimensional quantities, $\alpha^{\prime}|\tau|^{b}\left(\tau-\lambda^{\prime} h_{x}\right)$. Here $\tau$ is the volumetric bed shear stress (measured in $\mathrm{m}^{2} \mathrm{~s}^{-2}$ ), and $\alpha^{\prime}$ is an empirical constant whose value reflects some properties of the sand; $\alpha$ depends on the exponent, $b$ (see Van Rijn 1993). For $b=1 / 2$, 
$\alpha^{\prime}$ can be estimated as $\alpha^{\prime}=8 \tilde{\gamma} /(g(s-1))\left[\mathrm{s}^{2} \mathrm{~m}^{-1}\right]$, where $s$ is the sand density divided by the water density and $\tilde{\gamma}$ is a number between 0.1 and 1 . Then, the characteristic morphological time scale can be found according to $\Delta T=\delta^{2} /\left(\alpha^{\prime}[\tau]^{3 / 2}\right)$, where $[\tau]$ is the typical shear stress. For $b=1 / 2$, it is possible to show that for the entire range of physically relevant parameters of the system, $\Delta T \gg 1 / \Omega$. Typically, $\Delta T \sim 2$ years (see Komarova \& Hulscher 2000) and $1 / \Omega \sim 2$ hours. For different values of the exponent $b$, this qualitative result still holds. Therefore, we can neglect the changes in sediment during one tidal period and use tidal averaging on the right-hand side of equation (1.2).

Next, we consider the role of parameter $\lambda=\lambda^{\prime} /[\tau]$ in the equation for sediment transport. Its inverse measures the relative dragging force exerted by the tide in comparison with gravity. $\lambda^{\prime}\left[\mathrm{m}^{2} \mathrm{~s}^{-2}\right]$ depends on the properties of the sediment and can vary significantly. An attempt to express $\lambda$ in terms of other parameters of the system was made in Komarova \& Hulscher (2000). Based on the work of Fredsoe (1974) and Bagnold (1956), the following expression was derived: $\lambda \approx 3 \Theta_{c 0} g(s-1) d /\left(2 \tilde{\gamma} \tan \phi_{s}[\tau]\right)$, where $\Theta_{c 0}=0.047$ is the critical Shields parameter, $\tan \phi_{s}=0.3$ is the friction angle (Van Rijn 1993; Dyer 1986), $s=\rho_{\text {sand }} / \rho_{\text {water }}=2.65,[\tau]=v_{0} U / \delta$ with typical values between $10^{-4}$ and $10^{-3} \mathrm{~m}^{2} \mathrm{~s}^{-2}$, and the grain size, $d$, varies approximately from $50 \mu \mathrm{m}$ to $2 \mathrm{~mm}$. Large $\lambda$ means that gravity plays an important role. Small $\lambda$ corresponds to stronger tides ( $\lambda$ is inversely proportional to the tidal strength, $[\tau])$. We choose the parameter $\lambda$ to be the stress parameter of the system. The physical range of values of $\lambda$ is approximately between $10^{1}$ and $5 \times 10^{2}$.

Finally, we discuss the range of values for the exponent $b$ in the sediment flux expression; $b$ measures how strongly the drag force depends on the bottom shear stress (see e.g. Dyer 1986). Usually values between $1 / 2$ and $5 / 2$ are used (for example, in Seminara \& Tubino 1992, values $b=1 / 2$ and $b=3 / 2$ were taken). In Schielen et al. (1993), values from $b=1$ to $b=6$ were investigated, and the result only weakly changed with $b$. In all of the above papers, the sediment was treated as bed load. Other authors associated different values of $b$ with different mechanisms of sediment transport (such as rolling, sliding, saltating or suspended load). Therefore, it is important that the present analysis is not sensitive to changes in $b$. It can be seen from figure 3 that the results only qualitatively (and weakly) depend on the choice of $b$. In this work we will take $b=2$.

\subsection{Summary of the model}

Now we are ready to present the dimensionless model where velocities are measured in terms of $U$, distances $(x, z$ and $h)$ in terms of $\delta$, hydrodynamical time, $t$, in terms of $1 / \Omega$ and morphological time, $T_{m}$, in terms of $\Delta T$. Let us define the parameter $R$ as $R=2 U /(\delta \Omega)$. The dimensionless bottom shear stress can be written as

$$
\tau=(1+v) \Psi_{z z}(z=h),
$$

and the system under consideration is

$$
\begin{gathered}
(1 / R)\left(2 \partial_{t}-\partial_{z}^{2}\right) \Psi_{z z}=(v / R) \Psi_{z z z z}-\Psi_{z} \Psi_{x z z}+\Psi_{x} \Psi_{z z z} \\
z=H / \delta: \quad \Psi_{z z}=0, \quad \Psi_{x}=0 \\
z=h: \quad \Psi_{z z}-S \Psi_{z}=0, \quad-\Psi_{x}=\Psi_{z} h_{x} \\
\frac{\partial h}{\partial T_{m}}=-\frac{\partial}{\partial x}\left\langle\left|\tau_{b}\right|^{b}\left(\tau_{b}-\lambda h_{x}\right)\right\rangle .
\end{gathered}
$$



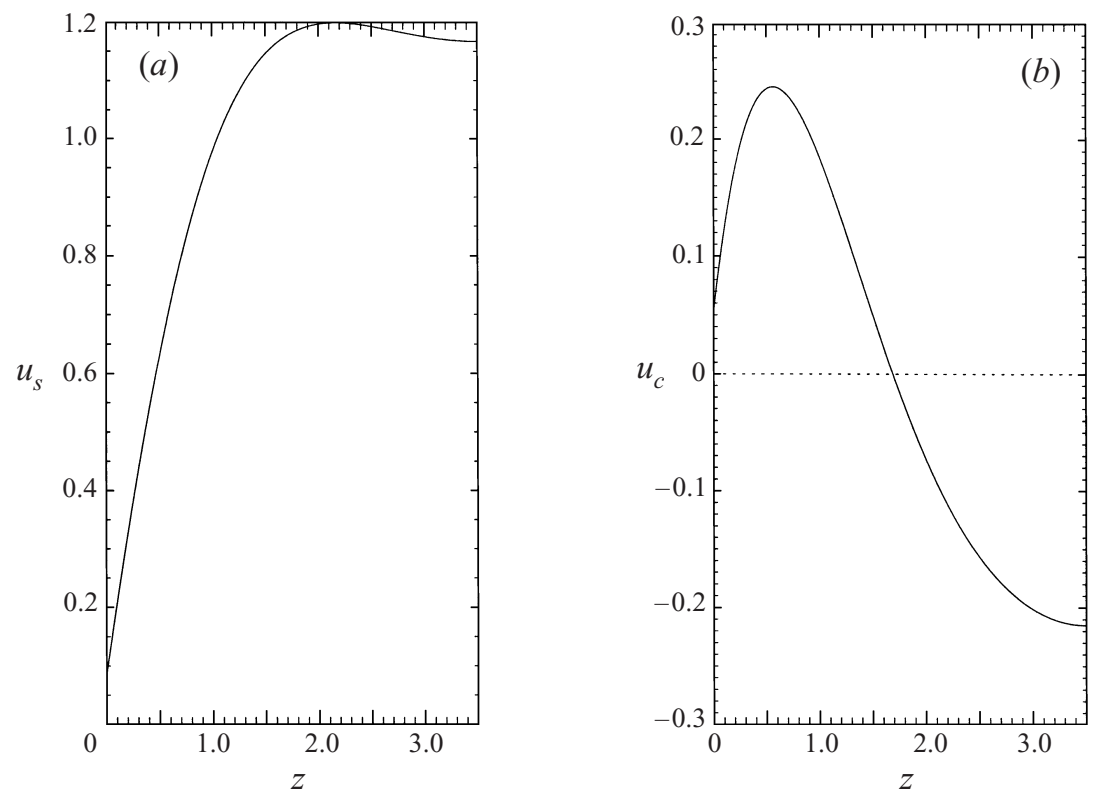

Figure 4. The two components of the tidal solution for $H / \delta=3.5, S=15$.

\section{Linear analysis}

\subsection{Tidal solution}

System (2.5-2.8) allows a solution corresponding to the unidirectional $M_{2}$-tide over a flat bed (the principal lunar semi-diurnal tide). Recall that we replace the effect of turbulent eddies by a bulk eddy viscosity and assume our basic state to be an (average) laminar tidal motion. This motion only takes place in the horizontal plane, i.e. $\Psi$ is a function of $z$ and $t$ only, and $h=v=0$. We do not assume any time-independent currents so that the tidal velocity, $u_{0}$, is

$$
u_{0}(z, t)=u_{s}(z) \sin t+u_{c}(z) \cos t .
$$

The analytical expression for $u_{0}$ can be written in the form

$$
u_{0}=\frac{\mathrm{i} H}{2 \delta} \frac{\cosh (1+\mathrm{i})\left(\frac{H}{\delta}-z\right)-\cosh (1+\mathrm{i}) \frac{H}{\delta}-\frac{1+\mathrm{i}}{S} \sinh (1+\mathrm{i}) \frac{H}{\delta}}{\frac{H}{\delta}\left(\frac{1+\mathrm{i}}{S}-\frac{1}{1+\mathrm{i}}\right) \sinh (1+\mathrm{i}) \frac{H}{\delta}+\frac{H}{\delta} \cosh (1+\mathrm{i}) \frac{H}{\delta}} \mathrm{e}^{\mathrm{i} t}+\text { c.c. }
$$

and the components $u_{s}$ and $u_{c}$ are shown in figure $4(a, b)$. Note that when solving the system for $u_{0}$, one needs to apply a normalization condition so that the solution is unique. The condition fixes the tidal strength,

$$
\frac{\delta}{H} \int_{0}^{H / \delta} u_{0}(z, t) \mathrm{d} z=\sin t,
$$

which means that the average tidal velocity (in dimensional units) looks like $U \sin (\Omega t$ ).

\subsection{Linear analysis and the soft mode}

Any state of the system can be characterized by the functions $\Psi\left(x, z, t, T_{m}\right)$ and $h\left(x, T_{m}\right)$. We start by perturbing the basic-state solution, $\Psi=\Psi_{0}(z, t), h=0$ (where 
$\left.\Psi_{0 z} \equiv u_{0}\right)$. Since the solution $\Psi_{0}$ does not depend on $x$, we can expand the perturbation in terms of plane waves $\mathrm{e}^{\mathrm{i} k x}$. The dependence on the slow time, $T_{m}$, can be also taken as $\mathrm{e}^{\mathrm{i} \omega T_{m}}$. Let us introduce a small parameter, $\epsilon \ll 1$. In the linear analysis, $\epsilon$ just measures how small the perturbation is with respect to the basic solution. The precise relation of $\epsilon$ with other parameters of the system will be defined later. The perturbed solution is

$$
\begin{aligned}
& \Psi(x, z, t)=\Psi_{0}(z)+\epsilon h_{1}\left(\mathrm{e}^{\mathrm{i}\left(k x+\omega T_{m}\right)} \Psi_{1}(z, t)+\text { c.c. }\right), \\
& h=\epsilon h_{1} \mathrm{e}^{\mathrm{i}\left(k x+\omega T_{m}\right)}+\text { c.c., }, \quad v=\epsilon v_{1} \mathrm{e}^{\mathrm{i}\left(k x+\omega T_{m}\right)}+\text { c.c. }
\end{aligned}
$$

where $\epsilon h_{1}$ is the (real) constant amplitude of the perturbation. For simplicity of notation we will avoid writing the $k$-dependence of $\Psi_{1}$ explicitly. For the general form (2.1), the correction to the turbulent viscosity, $v_{1}$, is given by $v_{1}=\alpha_{1}+\mathrm{i} k \alpha_{2} \Psi$. For Model I, the linearized viscosity response is conveniently expressed in terms of the stream function, $v_{1}=\tilde{u}^{-2} \Psi_{0 z} \Psi_{1 z}$, where we use the notation $\tilde{u} \equiv \sqrt{u_{s}^{2}(0)+u_{c}^{2}(0)}$. In the analysis below we used Model I. Calculations are very similar for the general model (2.1). We linearize the problem around the tidal solution to obtain the following system:

$$
\mathscr{L}_{k} \Psi_{1}=0,
$$

with the linear operator acting on the Fourier mode corresponding to $k$,

$$
\mathscr{L}_{k}=\frac{1}{R}\left(2 \partial_{t}-\partial_{z}^{2}\right) \partial_{z}^{2}-\frac{\partial_{z}^{4} \Psi_{0}}{\left(R \tilde{u}^{2}\right)}\left[\Psi_{0 z} \partial_{z} \cdot\right]_{z=0}+\mathrm{i} k \Psi_{0 z} \partial_{z}^{2}-\mathrm{i} k \partial_{z}^{3} \Psi_{0},
$$

where the dot means that the function $\Psi_{1}$ must be inserted. The linearized boundary conditions are

$$
\begin{gathered}
\left(\partial_{z}^{2}-S \partial_{z}\right) \Psi_{1}+\left.\partial_{z}\left(\partial_{z}^{2}-S \partial_{z}\right) \Psi_{0}\right|_{z=0}=0, \\
\mathrm{i} k \Psi_{1}+\left.\mathrm{i} k \partial_{z} \Psi_{0}\right|_{z=0}=0, \\
\left.\partial_{z}^{2} \Psi_{1}\right|_{z=H / \delta}=0, \\
\left.\mathrm{i} k \Psi_{1}\right|_{z=H / \delta}=0 .
\end{gathered}
$$

Owing to our choice of the tidal amplitude, the total flow of water must be equal to $\sin t$ :

$$
\frac{\delta}{H} \int_{h}^{H / \delta} \overline{\Psi(x, z, t)} \mathrm{d} z=\sin t,
$$

where the overbar means averaging in the $x$-coordinate. Because of expression (3.3), this condition is automatically true for all the harmonics of $\Psi_{1}$ with $k \neq 0$. For $k=0$, we have an extra condition:

$$
\left.\Psi_{1}\right|_{z=H / \delta}-\left.\Psi_{1}\right|_{z=0}=\left.\Psi_{0 z}\right|_{z=0}, \quad k=0
$$

(this follows from expression (3.3)). In this analysis, we use a time-truncation procedure in order to reduce partial differential equation (3.6) to a system of ordinary differential equations (see De Swart \& Zimmerman 1993; Hulscher et al. 1993; and Gerkema 1999 for justification of this method; in particular, in the recent work of Gerkema 1999, the effects of such truncation were investigated explicitly for sand waves, the difference between exact and truncated solutions was calculated, and it was demonstrated that truncation did not introduce any qualitative changes in the behaviour). We will only keep the first two time harmonics for every function of $t$, so for instance, $\Psi_{1}(t, z)=$ $\mathrm{i} \Psi_{1,0}(z)+\Psi_{1, s}(z) \sin t+\Psi_{1, c}(z) \cos t$. For $k \neq 0$, system (3.6)-(3.11) becomes a fourthorder differential equation in $z$ with four non-homogeneous boundary conditions. 
The linear operator is not singular, so a unique solution can always be found. For $k=0$, the situation is different. Conditions (3.9) and (3.11) do not exist anymore, and instead of them there is condition (3.13). This means that solutions with $k=0$ are determined up to a constant (which is not a problem, because the stream function only gives physically relevant quantities upon differentiation). Also one can see that solutions of system (3.6)-(3.11) with $k \neq 0$ in the limit $k \rightarrow 0$ satisfy equation (3.13), i.e. a mean flow solution can be obtained as a limit of solutions with a finite $k$.

The sediment response (where we set $b=2$ ) gives

$$
\mathscr{D} \equiv-\mathrm{i} k\left\langle\Psi_{0 z z}^{2}\left(3 \Psi_{1 z z}+\left(1 / \tilde{u}^{2}\right) \Psi_{0 z} \Psi_{0 z z} \Psi_{1 z}-\mathrm{i} k \lambda+\Psi_{0 z z z}+\Psi_{0 z z}\right)\right\rangle_{z=0}-\mathrm{i} \omega(k)=0 .
$$

Because of the specific time dependence of $\Psi_{0 z}$, namely $\Psi_{0 z}=u_{s} \sin t+u_{c} \cos t$, the last two terms in the angular brackets are identically zero. It is also easy to show that all the terms in equation (3.14) coming from the time averaging of the water flow are real. Therefore, $\mathrm{i} \omega(k)$ is also real (i.e. there are no travelling waves). This can be explained using a symmetry argument: because the perturbation is chosen to be $\propto \cos k x$, there is no preferred direction in the system, and therefore all terms associated with moving in the $x$-direction must be zero. We will use this fact to introduce the following notation:

$$
\mathrm{i} \omega(k) \equiv \Gamma(k),
$$

where the growth rate, $\Gamma$, is a real function of $k$ and is given in figure 3 . The linear analysis of problem (3.6)-(3.11) shows that the behaviour of the system qualitatively depends on the parameter $\lambda$. We find that when the ratio of dragging force to gravity (as measured by $1 / \lambda$ ) exceeds a certain value, $1 / \lambda_{c}$, a finite bandwidth of modes about a wavenumber, $k_{c}$, becomes unstable. Note that in the linear analysis, the amplitude $\epsilon h_{1}$ of the solution is undetermined. The flow response is proportional to the amplitude of the bed perturbation, which is arbitrary.

Problem (3.6)-(3.11) contains a homogeneous differential equation and is only driven through the (inhomogeneous) boundary conditions. If we define

$$
\tilde{\Psi}_{1}=\Psi_{1}+a_{0}+a_{1} z+a_{2} z^{2}+a_{3} z^{3},
$$

then constants $a_{j}, 0 \leqslant j \leqslant 3$, can be found such that the boundary conditions for $\tilde{\Psi}$ become homogeneous, whereas the differential equation acquires a right-hand side, $\mathscr{F}$,

$$
\mathscr{L}_{k} \tilde{\Psi}_{1}=\mathscr{F} .
$$

This formulation is more convenient for the weakly nonlinear analysis.

From equation (3.14) one can explicitly see the obvious result that $\Gamma(k=0)=0$, the existence of a soft mode. The solution $\Psi_{1}$ corresponding to the soft mode is non-trivial. Its amplitude is determined by an arbitrary amplitude of the bottom perturbation with $k=0$. This solution corresponds to the flow response to the uniform lift of the bottom, $h$. This mode is neutrally stable in the linear analysis, but it plays an important role if we take nonlinearity into account. It is called the mean flow (we will denote it as $\Psi_{\text {mean }}$ ) and participates in the nonlinear interaction through coupling with the bandwidth of unstable modes. The whole effect of the mean flow has been overlooked so far. We shall include it in the analysis below and show that it can be responsible for sand bank formation. We emphasize that the mean flow mode describes local mean elevations (depressions) of the bed; the global average elevation is zero, which is consistent with (1.2). 


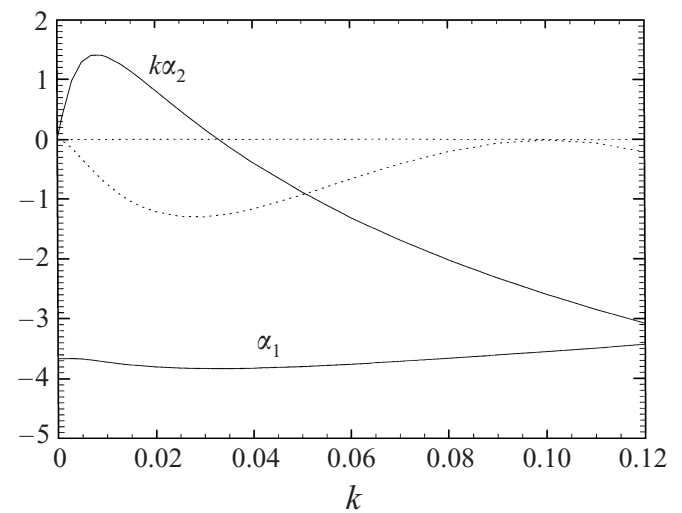

FIGURE 5. The coefficients $\alpha_{1,2}$ as functions of $k . H=45 \mathrm{~m}, v_{0}=0.006 \mathrm{~m}^{2} \mathrm{~s}^{-1}, S=5$, and the dotted line is the growth rate curve magnified by a factor of 200 .

\subsection{The role of viscosity parameterization}

For the linear analysis described above, we used viscosity Model I and obtained the growth rate curve given in figure 3 . In this case, the coefficients $\alpha_{1,2}$ of formula (2.1) are expressed in terms of the harmonics of the stream function:

$$
\alpha_{1}=\left.\tilde{u}^{-2}\left(u_{s} \partial_{z} \Psi_{1, s}+u_{c} \partial_{z} \Psi_{1, c}\right)\right|_{z=0}, \quad \alpha_{2}=-2 /\left.\left(k \tilde{u}^{2}\right) \partial_{z} \Psi_{1,0}\right|_{z=0}
$$

and their dependence on $k$ is presented in figure 5. This model leads to finite values of $k_{c}$, and $2 \pi / k_{c}$ has size similar to the typical wavelength of sand waves.

We also performed linear analyses for other forms of viscosity parameterization (2.1). The results can be generalized in the following way.

(a) If $\alpha_{1} \geqslant 0$ for small values of $k$, the mechanism for damping very long waves is absent, and in supercritical conditions, all wavelengths from some finite number up to infinity are excited. The situation $\alpha_{1}>0$ corresponds to the eddy viscosity whose time average is smaller in the troughs and larger over the crests of sand waves.

(b) If $\alpha_{1}$ is finite and negative for small values of $k$, the system chooses a finite most excited wave number, and the ultra-long waves are damped. In this case, the eddy viscosity is larger in the troughs than it is over the crests. Note that for Model I, coefficient $\alpha_{1}$ (given by expression (3.18)) is finite and negative, see figure 5 .

The explanation is as follows. Let us start with a constant-viscosity model, i.e. $\alpha_{1}=\alpha_{2}=0$. In this case, the mechanism we described in $\S 1.2$ for the formation of the circulating eddies which scrape the sand along the bed from trough to crest, holds for all wavelengths. It weakens as $k \rightarrow 0$ and indeed, near $k=0$, the instability growth rate $\Gamma(k)$ is positive and proportional to $k^{2}$. If the tidal strength is enough to overcome gravity, the growth rate curve has a positive gain band for a range of $k, 0<k<k_{0}$, i.e. all wavelengths starting from $2 \pi / k_{0}$ and up to infinity are excited (shorter waves are damped by the gravity term).

This is not what is observed. Sand waves seem to have a preferred wavelength between 200 and $800 \mathrm{~m}$ and coexist with sand banks which are 2-10 km long (Huntley et al. 1993). In other words, the power spectrum of the bed consists of wavelengths typical for sand waves with some energy in a small range near $k=0$. This is consistent with the growth rate shown in figure 3 when one adds in the nonlinear mechanism for driving long waves by short ones.

When one takes account of viscosity variations as in formula (2.1), and the coeffi- 


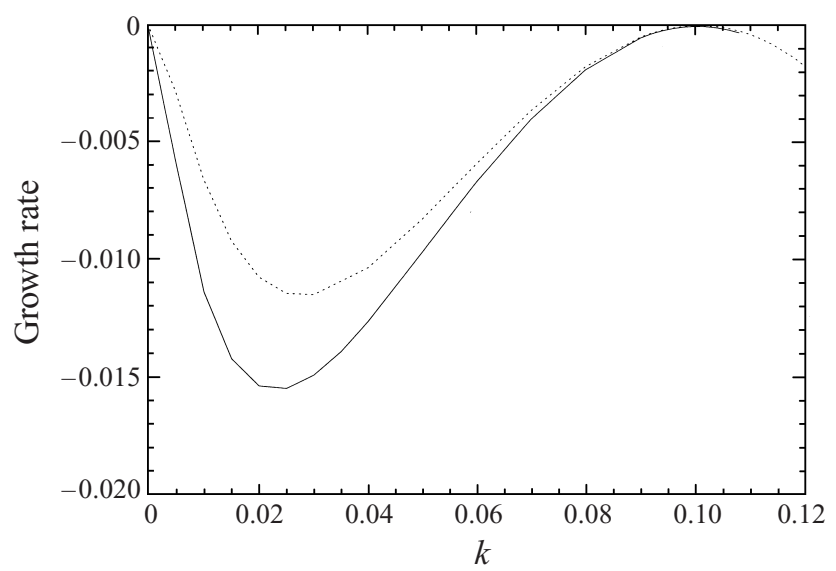

FIGURE 6. The linear growth rate curves for the parameter set as in figure 4; the dotted line corresponds to viscosity Model I, the solid line to Model II with $\alpha_{1}=-4.1, \alpha_{2}=0$. In both cases, the critical wavelength is $528.8 \mathrm{~m}$.

cient $\alpha_{1}$ is finite and negative for small values of $k$, there is a competing mechanism for driving the circulating eddies of figure 2 which counteracts the sense of rotation. It has a similar origin to the cells discussed in $\S 1.2$ in that the interaction of tidal direction and viscosity is asymmetric. What is very important is that for very small wavenumbers it produces a sense of rotation which weakens the destabilizing cellular motion sufficiently so that it no longer can overcome the stabilizing influence of gravity. This makes $\Gamma(k)$ negative for $k$ near zero (see figure 3).

Finally, if $\alpha_{1}$ is finite and positive for small values of $k$, the vortices of figure 2 are enhanced for long waves. Therefore, such viscosity model does not provide a wavelength selection mechanism.

What is encouraging is that these results are not very sensitive to the particular form of eddy viscosity parameterization. It turns out that the behaviour is very similar for Models I and II with $\alpha_{1}<0$. In fact, for the same set of physical parameters, it is always possible to find values for $\alpha_{1,2}$ such that the critical wavelength coincides with the one given by Model I. The coefficient $\alpha_{2}$ does not play a significant part because the corresponding term in expression (2.1) is small for small $k$. In figure 6 we present the growth rate curves, $\Gamma(k)$, for Models I and II. The physical parameters are the same in both cases. The biggest difference between the two functions $\Gamma(k)$ is that the curvature near $k=0$ is larger for Model II than it is for Model I.

\subsection{Summary of the preliminary results}

Now we are ready to perform a nonlinear analysis of the system. Before we begin, we would like to emphasize the features of the linear system essential for the nonlinear theory to be developed.

The growth rate curve for the values of the control parameter slightly after the bifurcation point has its absolute maximum separated from $k=0$ by a bandwidth of modes with negative values of the growth rate. The system chooses a first excited mode with a finite wavelength.

The $k$-dependence of the growth rate near $k=k_{c}$ is locally quadratic. The mode with $k=0$ is neutrally stable.

The $k$-dependence of the growth rate near $k=0$ is locally quadratic. 
The same holds for a number of systems considered by other authors. This includes the generation of sand ripples (Blondeaux 1990), alternate bars in straight channels (Komarova \& Newell 1995) and sand dunes in channels (Fredsoe 1974).

\section{Nonlinear analysis}

The linear analysis above predicts the formation of sand waves. Very long waves are linearly damped. Our goal is to explain the coexistence of sand waves and sand banks, which have a much larger wavelength. In order to do this, we take into account some effects of nonlinearity in the problem. We use a perturbative method assuming that the system is not too far above the threshold, and separate the fast and slow dynamics (see e.g. the review by Newell et al. 1993) to describe the growth and interaction of sand waves and sand banks. The procedure of nonlinear analysis used here is rather standard (see for instance Newell \& Moloney 1992, where a weakly nonlinear analysis for the Maxwell-Bloch system is developed, or Schielen et al. 1993, where a system for a vertically averaged channel flow is considered). We will describe the method in this section. The detailed calculations for each order are given in the Appendix.

We suppose that the control parameter, $\lambda$, is only slightly smaller than its critical value, i.e. $\left|\lambda-\lambda_{c}\right|=\lambda_{c} \epsilon^{2}$. We use this to define $\epsilon$. The fastest growing mode has wavelength $k_{c}$ (and corresponds to the sand waves), and the zero mode is neutrally stable. We start with the following general double expansion of the solution (where $\left.E_{c} \equiv \mathrm{e}^{\mathrm{i}\left(k_{c} x+\omega_{c} T_{m}\right)}\right)$ :

$$
\Psi=\sum_{n=0}^{\infty} \sum_{m \leqslant n} \epsilon^{n} E_{c}^{m} \Psi_{n(m)}(X, T, z, t)+\text { c.c., } \quad h=\sum_{n=1}^{\infty} \sum_{m \leqslant n} \epsilon^{n} E_{c}^{m} h_{n(m)}(X, T)+\text { c.c. }
$$

Note that in order to include the finite bandwidth (of width proportional to $\epsilon$ ) of unstable modes, the coefficients of $E_{c}^{m}$ in this expansion are allowed to depend on slow time and space variables, $X=\epsilon x$ and $T=\left(T_{1}, T_{2}, \ldots\right)$ with $T_{1}=\epsilon T_{m}, T_{2}=\epsilon^{2} T_{m}, \ldots$ Let us denote the solution of system (3.6)-(3.11) with $k=k_{c}$ as $\Psi_{c}$. We will write down the first few terms of this expansion explicitly:

$$
\begin{aligned}
\Psi= & \frac{1}{2} \Psi_{0}(z, t)+\epsilon A(X, T) E_{c} \Psi_{c}(z, t)+\epsilon^{2}\left(\frac{1}{2} \Psi_{2(0)}(X, T, z, t)\right. \\
& \left.+E_{c} \Psi_{2(1)}(X, T, z, t)+E_{c}^{2} \Psi_{2(2)}(X, T, z, t)+\frac{1}{2} B(X, T) \Psi_{\text {mean }}(z, t)\right)+ \text { c.c. }+\ldots, \\
& \\
h= & \epsilon A(X, T) E_{c}+\epsilon^{2}\left(h_{2(1)}(X, T) E_{c}+h_{2(2)}(X, T) E_{c}^{2}+\frac{1}{2} B(X, T)\right)+\text { c.c. }+\ldots
\end{aligned}
$$

The first term on the right-hand side of equation (4.2) is the basic-state solution representing a tide over a flat bottom. The second term is the solution of the linear problem corresponding to the fastest growth rate. The envelope $A$ (in the previous section we denoted it as $h_{1}$ ) is allowed to vary slowly in time and space to incorporate finite bandwidth effects. The next three terms on the right are second harmonics which appear as a result of the nonlinearity. Their dependence on the slow variables will be determined shortly. Finally, the term proportional to $B$ on the right-hand side is the solution of the linear problem for the $k=\omega=0$ mode, corresponding to the bed distortion amplitude $B$. Note that the component of the $h$-expansion corresponding 
to $\epsilon^{2} h_{2(0)}$ can be taken to be zero if we impose the condition that the amplitude $B$ averaged over $X$ is zero (sand mass conservation).

The goal is to find a system of partial differential equations describing the nonlinear dynamics of the slow varying envelopes, $A$ and $B$. This system will explain the mechanism of generation of sand waves and tidal banks on the tidal background. In order to find such a system, we will consider the equations at different orders of $\epsilon$. At certain orders, the system becomes over-determined and we will need to impose some kind of solvability condition to make the equations self-consistent. This solvability condition gives us asymptotic expansions for $\partial A / \partial T_{m}\left(=\epsilon \partial A / \partial T_{1}+\epsilon^{2} \partial A / \partial T_{2}+\ldots\right)$ and $\partial B / \partial T_{m}$ which govern the slow dynamics of the system.

\subsection{General consideration}

We will call the mode proportional to $\epsilon^{n} E_{c}^{m}$ the $(n m)$-mode. The corresponding bed distortion component looks like $\epsilon^{n} h_{n(m)} \mathrm{e}^{m i\left(k_{c} x+\omega_{c} T_{m}\right)}$. Using expressions (4.2)-(4.3) in system (2.5)-(2.8), we can write down the contributions at the $(\mathrm{nm})$-order as

$$
\begin{aligned}
& \mathscr{L}_{k=m k_{c}} \Psi_{n(m)}=F_{n l}, \\
& \left(\partial_{z}^{2}-S \partial_{z}\right) \Psi_{n(m)}+\left.\partial_{z}\left(\partial_{z}^{2}-S \partial_{z}\right) \Psi_{0} h_{n(m)}\right|_{z=0}=C_{n(m)}, \\
& \mathrm{i} m k_{c} \Psi_{n(m)}+\left.\mathrm{i} m k_{c} \partial_{z} \Psi_{0} h_{n(m)}\right|_{z=0}=D_{n(m)}, \\
& \left.\partial_{z}^{2} \Psi_{n(m)}\right|_{z=H / \delta}=0, \\
& \left.\operatorname{i} m k_{c} \Psi_{n(m)}\right|_{z=H / \delta}=0 \text {, } \\
& -\mathrm{i} m k_{c}\left\langle\Psi_{0 z z}^{2}\left(\left(3 \partial_{z}+\frac{1}{\tilde{u}^{2}} \Psi_{0 z} \Psi_{0 z z}\right) \partial_{z} \Psi_{n(m)}-\mathrm{i} m k_{c} \lambda_{c}\right)\right\rangle_{z=0}-\mathrm{i} m \omega_{c} h_{n(m)}=\mathscr{G}_{n(m)},
\end{aligned}
$$

where $C_{n(m)}$ and $D_{n(m)}$ do not depend on $z$ and contain information about the boundary conditions related to the previous $(<n)$ orders in $\epsilon, F_{n l}$ comes from the corresponding nonlinear terms and $\mathscr{G}_{n(m)}$ reflects the effect of nonlinearities and slow variations. Note that all the terms in equation (4.9) are functions of slow coordinates only. This is because this equation comes from the sediment conservation law (2.8), where the fast time dependence is eliminated by averaging over a tidal period, and all functions are evaluated at the point $z=h$. For $m=0$ modes, there is also a normalization restriction,

$$
\int_{0}^{H / \delta} \partial_{z} \Psi_{n(0)} \mathrm{d} z=\gamma_{n},
$$

where $\gamma_{n}$ are constants which come from previous harmonics. This latter condition guarantees that the perturbation produces no net tidal motion.

Note that the system is over-determined, because five boundary conditions are imposed on a fourth-order ordinary differential equation. The requirement of selfconsistency will enable us to find conditions on the unknown envelopes.

The general procedure of solving system (4.4)-(4.8) at each order $(\mathrm{nm})$ is as follows. First we rewrite equations (4.4)-(4.7) using the time-series truncation, thus obtaining a system of three fourth-order partial differential equations in $z$ for the unknown functions $\Psi_{n(m), 0}, \Psi_{n(m), s}$ and $\Psi_{n(m), c}\left(\right.$ where $\left.\Psi_{n(m)}=\Psi_{n(m), 0}+\Psi_{n(m), s} \sin t+\Psi_{n(m), c} \cos t\right)$. Note that at the stage $(\mathrm{nm})$, we need to have obtained the necessary modes at orders less that $n$. These modes are used to evaluate $F_{n l}, C_{n(m)}$ and $D_{n(m)}$. Then, we numerically solve the resulting inhomogeneous boundary value problem using the 
so-called shooting to a fitting point method. Finally, we substitute the flow solution into the sediment transport equation, (4.9).

Before we explicitly list the solvability conditions, we would like to give one example of system (4.4)-(4.8). For the mode (11) it is given by equations (3.6)-(3.11), (3.14). There, $m=n=1, h_{1(1)}=1, F_{1(1)}=C_{1(1)}=D_{1(1)}=\mathscr{G}_{1(1)}=0$ and the solution is just $\Psi_{c}$. The last equation defines the critical value of $\omega_{c}$ (which is zero by the choice of $k=k_{c}, \lambda=\lambda_{c}$ ). The solution at order (11) reproduces the linear analysis of $\S 2$.

For higher orders, the terms coming from nonlinear interactions and slow behaviour become non-trivial. The solution in each order $(\mathrm{nm})$ can be represented as a sum of the solution of the corresponding homogeneous system and a particular solution of the non-homogeneous system, i.e.

$$
\Psi_{n(m)}=\Psi_{1}\left(k=m k_{c}\right) h_{n(m)}+\Psi_{n(m)}^{n l},
$$

where the $\Psi_{1}\left(k=m k_{c}\right)$ is the solution of system (3.6)-(3.11) with $k=m k_{c}$, and the $\Psi_{n(m)}^{n l}$ part is driven by the nonlinearities and slow derivatives. We will substitute solution (4.11) into equation (4.8) and make sure that the equation is satisfied. There are three cases:

(a) $m=0$ : the solvability condition is

$$
\mathscr{G}=0,
$$

and the amplitude $h_{n(0)}$ is undetermined. It means that at every order of $\epsilon$ we will get a solution of the form

$$
\Psi_{n(0)}=\Psi_{\text {mean }} h_{n(0)}+\Psi_{n(0)}^{n l} .
$$

(b) $m=1$ : solution $\Psi_{1}\left(k=k_{c}\right) \equiv \Psi_{c}$ makes the left-hand side of equation (4.8) zero (by the definition of the $\omega_{c}$ ). The solvability condition is

$$
-\mathrm{i} k_{c}\left\langle\Psi_{0 z z}^{2}\left(3 \partial_{z}+\frac{1}{\tilde{u}^{2}} \Psi_{0 z} \Psi_{0 z z}\right) \partial_{z} \Psi_{n(1)}^{n l}\right\rangle_{z=0}-\mathscr{G}_{n(1)}=0 .
$$

The amplitude $h_{n(1)}$ remains undetermined and $h_{n(1)} \Psi_{c}$ is a solution of the system for each $n$.

(c) $m>1$ : the solution $h_{n(m)} \Psi_{1}\left(k=m k_{c}\right)$ has the amplitude determined uniquely by equation (4.9) (modulo the remarks in the end of this paper about sub- and super-harmonics). There is no other solvability condition.

Without loss of generality, we can take $h_{n(1)}=0$ for all $n>1$ and $h_{n(0)}=0$ for all $n>2$. The corresponding solutions are proportional to the eigenvectors which we introduce at orders $\epsilon$ and $\epsilon^{2}$ with the amplitudes $A$ and $B$ respectively. The amplitudes of those higher-order solutions can be included into the the envelopes $A$ and $B$. By introducing non-trivial corrections $h_{n(1)}$ and $h_{n(0)}$ we would just redefine $A$ and $B$.

Note that the procedure described here is slightly different from the conventional one (see e.g. Schielen et al. 1993). Normally, one has a singular linear operator and the solvability condition is given by the Fredholm alternative theorem, which ensures that one can solve for iterates. In our case, the linear operator (in the formulation used here) is not singular (note the presence of the driving terms proportional to $h_{n(m)}$ in equations (4.5)-(4.6)). System (4.4)-(4.8) always has a solution. However, the problem is still overdetermined because of the conservation law for sand. Equation (4.9) itself becomes the solvability condition for this system. It results in certain (nonlinear) partial differential equations for the envelopes. However, if we remember that the result of the linear hydrodynamical equations is nothing but an expression $\Psi=\Psi[h]$, i.e. the flow as a linear functional of the bed deformations, we can formally rewrite 
the problem at each order as a non-homogeneous equation $\mathscr{M} h=\mathscr{R}$, where $\mathscr{M}$ is a singular linear operator. Then the solvability conditions described above can be derived from the corresponding Fredholm alternative theorem.

\subsection{Results}

We refer the reader to the Appendix for the details of the nonlinear analysis. In this study, it is necessary to go to fourth order in $\epsilon$ to recover non-trivial dynamics of the amplitude $B$. At each order, we find the corresponding components in expansions (4.2)-(4.3) and a solvability condition for the envelopes, $A$ and $B$. The results are

$$
\begin{gathered}
A_{T_{1}}=B_{T_{1}}=0, \\
A_{T_{2}}=\sigma / \lambda_{c} A+\gamma \partial_{X}^{2} A+c|A|^{2} A+\eta A B, \\
B_{T_{2}}=\beta \partial_{X}^{2} B+\xi \partial_{X}^{2}|A|^{2},
\end{gathered}
$$

where $\sigma, \gamma, c, \eta, \beta$ and $\xi$ are real constants.

\subsection{Remarks}

The nonlinear analysis was performed for both eddy viscosity Model I and Model II (with $\alpha_{1}<0$ and $\alpha_{2}=0$ ). The resulting nonlinear system for the envelopes (4.15)(4.17) is qualitatively the same for both cases. The values of the coefficients have the same order of magnitude and sign.

Let us examine system (4.15)-(4.17). Equation (4.15) tells us that the instability is a pitchfork and not a Hopf bifurcation. There is no group velocity. Migration of sand wave packets would require a unidirectional component in the tide or some other influence that breaks the $x \rightarrow-x$ symmetry.

Next, the coefficients in front of linear terms in system (4.16)-(4.17) can be obtained from the growth rate curve as well as directly from equations in the corresponding orders. Namely,

$$
\begin{aligned}
& \sigma=\lambda_{c}\left[\frac{\partial \Gamma}{\partial(-\lambda)}\right]_{c}, \\
& \gamma=-\frac{1}{2}\left[\frac{\partial^{2} \Gamma}{\partial k^{2}}\right]_{c}, \\
& \beta=-\frac{1}{2}\left[\frac{\partial^{2} \Gamma}{\partial k^{2}}\right]_{0},
\end{aligned}
$$

where the subscript $c$ means 'critical' $\left(\lambda=\lambda_{c}, k=k_{c}\right)$ and the subscript 0 means $\lambda=\lambda_{c}, k=0$. The coefficients $\sigma, \gamma$ and $\beta$ are real.

The coefficients $c, \eta$ and $\xi$ are also real numbers. The corresponding analytical expressions are not presented here because they contain dozens of terms. We just point out that they are obtained by averaging (over a tidal period) algebraic functions of modes $\Psi_{n(m)}(z=0)$ and their $z$-derivatives. These coefficients also directly depend on $k_{c}$ and $\lambda_{c}$.

Finally, we can rewrite system (4.15)-(4.17) using more natural variables. To start, recall that $\partial_{T_{m}}=\epsilon \partial_{T_{1}}+\epsilon^{2} \partial_{T_{2}}$, which is equal to $\epsilon^{2} \partial_{T_{2}}$ because of equations (4.15). Next, we note that in the analysis above, we scaled all distances (horizontal and vertical) with $\delta$, the turbulent boundary layer thickness. It is far more appropriate to scale horizontal distances with the wavelength of sand waves, $L$ (which is much larger than $\delta$ ). Similarly, it is convenient to measure the vertical bed distortions in terms of a typical sand wave height, $l$ (which is smaller than $\delta$ ). According to this, 


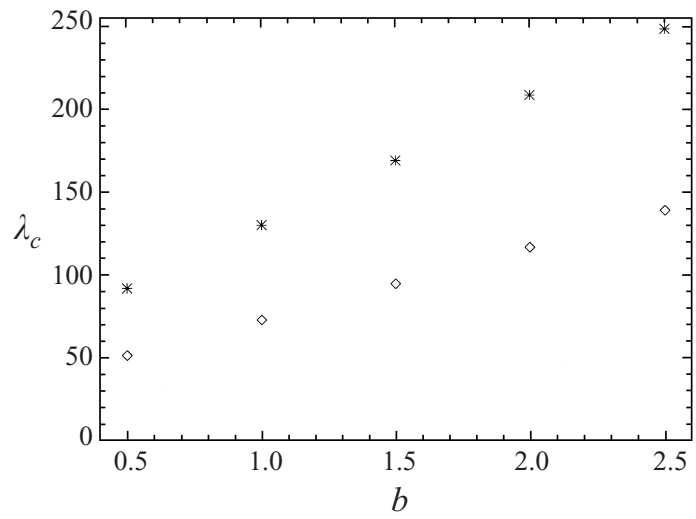

FiguRE 7. The critical control parameter for different values of $b$. Stars correspond to (i) $H=30 \mathrm{~m}$, $v_{0}=0.006 \mathrm{~m}^{2} \mathrm{~s}^{-1}, S=15$, diamonds correspond to (ii) $H=45 \mathrm{~m}, v_{0}=0.009 \mathrm{~m}^{2} \mathrm{~s}^{-1}, S=12$.

let us set $\mathscr{A}=\epsilon A \delta / l, \mathscr{B}=\epsilon^{2} B \delta / l$ and $\tilde{x}=x \delta / L$. Now we can incorporate all the solvability conditions obtained in this section into a system of two partial differential equations with constant coefficients:

$$
\begin{gathered}
\mathscr{A}_{T_{m}}=\sigma\left(\lambda_{c}-\lambda\right) / \lambda_{c} \mathscr{A}+\gamma \mathscr{A}_{\tilde{x} \tilde{x}}+c|\mathscr{A}|^{2} \mathscr{A}+\eta \mathscr{A} \mathscr{B}, \\
\mathscr{B}_{T_{m}}=\beta \mathscr{B}_{\tilde{x} \tilde{x}}+\xi|\mathscr{A}|_{\tilde{x} \tilde{x}}^{2}
\end{gathered}
$$

(remember that in order to be supercritical, $\lambda$ must be less than $\lambda_{c}$; hence $\sigma$ in equation (4.18) and the first term in the right-hand side of equation (4.21) are both positive). In the rest of this paper we used the following rescaling coefficients: $L=800 \mathrm{~m}$ and $l=2.5 \mathrm{~m} . T_{m}$ is measured in units of 2 years. Note that equation (4.22) is simply a normal form for the sediment transport equation when the bed deformation is approximately sinusoidal with flux $-\xi \partial_{\tilde{x}}|\mathscr{A}|^{2}-\beta \partial_{\tilde{x}} \mathscr{B}$.

\section{Discussion and estimates}

Here we will discuss the sensitivity of the weakly nonlinear stability analysis (system (4.21)-(4.22)) to the choice of the physical parameters $b, v_{0}, H, S$ and $R$ since they are either phenomenological (i.e. chosen on empirical grounds) or their range is large. We follow this with a discussion of the coefficients $\sigma, \gamma, c, \eta, \beta$ and $\xi$ in equations (4.21)-(4.22), their typical sizes and dependence on the physical parameters (in particular, on $S$ and $H / \delta$ ). We will also comment on how the results depend on the choice of the eddy viscosity model. Next, we investigate the predictions of the equations themselves and present the main result, namely that sand banks are a direct consequence of variations in sand wave intensity. We follow this with several further remarks concerning standard properties of equations (4.21)-(4.22) and end the section with a suggestion that when $1 / \lambda$ is strongly supercritical, one can expect period doubling to occur.

\subsection{Physical parameters}

First of all we will comment on the role played by the parameter $b$, the power law in the sediment flux parameterization. It has been observed that, if the rest of the physical parameters are fixed and only the power $b$ changes, the predicted wavelength of the sand waves stays the same, and only the critical value of the control parameter, 
$\lambda_{c}$, changes. In figure 7, we present some examples of $b$ and the corresponding values of the critical $\lambda$. The estimates are given for two sets of physical parameters: (i) $H=30 \mathrm{~m}, v_{0}=0.006 \mathrm{~m}^{2} \mathrm{~s}^{-1}, S=15$ and (ii) $H=45 \mathrm{~m}, v_{0}=0.009 \mathrm{~m}^{2} \mathrm{~s}^{-1}, S=12$. The critical wavelength for the systems (i) and (ii) are 894.9 and $636.1 \mathrm{~m}$ respectively. The result that $k_{c}$ does not depend on the value of $b$, is shown in figure 3 . There, we plotted the growth rate curve for five values of $b$ (parameter set (i)). For each value of $b$, we used $\lambda=99 \% \lambda_{c}$. It can be also observed that as $1 / \lambda$ is slightly supercritical, the band width of modes with a positive growth rate does not depend on the concrete value of $b$ (growth rate curves corresponding to different $b$ cross zero at the same two points). This means that $b$ does not define the slow $x$-variation of the excited mode. This is rather encouraging because formula (2.8) is purely empirical, and a strong dependence on the value of $b$ would throw the analysis into question. Because of the weak dependence on $b$, we choose its value for analytical convenience. In particular, we choose it so that the sediment flux has a Taylor series expansion about the basic tidal solution. This is only possible if $b$ is an even integer, and the only one that falls into the physically realistic range is $b=2$.

The last remark concerning the choice of $b$ is related to the corresponding values of the critical control parameter. The fact that $\lambda_{c}$ increases (linearly) with $b$ (see figure 7 ) is not surprising. The bigger the exponent in the sediment flux expression is, the more strongly the flux depends on the shear stress and the harder it becomes for the gravity force to balance the 'scraping' term. Since $\lambda$ is an empirical parameter whose meaning is only known to us qualitatively, we will choose it to be somewhat below its calculated critical value to ensure the excitation of sand waves.

The rest of parameters involved in the system are chosen to fall in a physically realistic range. Typical values for $U$ are about $1 \mathrm{~m} \mathrm{~s}^{-1}$, the tidal frequency is $\Omega=$ $1.4 \times 10^{-4} \mathrm{~s}^{-1}$, and the average eddy viscosity is of the order $10^{-3}-10^{-2} \mathrm{~m}^{2} \mathrm{~s}^{-1}$ (see e.g. Pedlosky 1987). This corresponds to values of $c_{1}$ approximately between $10^{-4}$ and $10^{-3}$. The typical turbulent boundary layer thickness is then about $10 \mathrm{~m}$. Sand waves have been observed in shallow seas with the depth of about $20-45 \mathrm{~m}$. This gives the range for the dimensionless ratio $H / \delta$ of 2-4.5. The parameter $R$ lies between 500 and 1000. In order to find estimates for the resistance parameter, $S=\delta C_{d} /\left(H c_{1}\right)$, we use the range for the drag coefficient between approximately $10^{-3}$ and $10^{-2}$ (see e.g. Maas \& van Haren 1987; Bowden 1983; Schielen et al. 1993; Soulsby 1990). This means that the order of magnitude for $S$ is roughly $10^{1}$.

\subsection{Typical values for coefficients in the envelope equations}

Here we will present the results for the coefficients in the envelope equations (4.21)(4.22). Only the dependence of the coefficients on $S$ and $H / \delta$ is discussed. The third dimensionless parameter present in system (2.5)-(2.8) is $R$, but it can be scaled out by redefining $x^{\prime}=x R$ and $\lambda^{\prime}=\lambda / R$. This is a consequence of the shallow-water approximation we are using here. The parameter $R$ was kept in equation (2.5) in order to follow the more traditional notation. In what follows we will fix $R=700$ which is a typical value. Model I for eddy viscosity is discussed here. See the next subsection for results for Model II.

$\sigma$ : this coefficient gives the linear growth rate of the sand waves per unit change of $\lambda$. Its typical values are $10^{-1}-1$ (see figure $8 a$ ). Physically this means that the system is not very sensitive to slight changes of the control parameter, $\lambda$. This is a reasonable result. It means that even if $\lambda$ differs from its critical value by $40-50 \%$, the product $\sigma\left(\lambda_{c}-\lambda\right) / \lambda_{c}$ is still a small number, i.e. we are still in a weakly nonlinear regime. The coefficient $\sigma$ increases both with $S$ and $H / \delta$. 
$\gamma$ : a typical value of the diffusion coefficient is $10^{-2}$ (see figure $8 b$ ). $\gamma$ is always positive, because the growth rate curve has a maximum at $k=k_{c}$, and $\gamma$ is proportional to its curvature there. The coefficient $\gamma$ defines the scale on which the sand waves amplitude, $\mathscr{A}$, varies. $\gamma$ grows with $S$.

$\beta$ : at $k=0$, the curvature of the growth rate relation is larger than it is at $k=k_{c}$ (see figure 3 ). This means that the diffusion coefficient in the $\mathscr{B}$-equation is relatively large (about 20 times larger than the diffusion coefficient for the sand wave equation, $\gamma$ ). The typical range of $\beta$ is $10^{-1}-1$, and $\beta$ increases both with $S$ and $H / \delta$ (see figure $8 c$ ). Note that this coefficient is positive because $k=0$ is a local maximum. This is a consequence of the turbulent viscosity model we are using. If instead of parameterization (2.1) with $\alpha_{1}<0$ we employed the usual $v_{t}=$ const model, the curvature of the growth rate curve near $k=0$ would have had a different sign. It was found in Hulscher (1996) that the super-long waves (modes with very small values of $k$ ) are always excited if the eddy viscosity is assumed to be a constant. Note that in this case the equation for $\mathscr{B}$ would look like

$$
\mathscr{B}_{T_{m}}=\beta_{1} \mathscr{B}_{x x}+\beta_{2} \mathscr{B}_{x x x x}+\xi^{\prime}|\mathscr{A}|_{x x}^{2}+\text { other nonlinearities, }
$$

with $\beta_{1}<0, \beta_{2}<0$ (this follows from the $k$-dependence of the growth rate near zero). The sign of coefficient $\xi^{\prime}$ will be discussed a little later. We have examined other modifications of dependence (2.2). For instance, higher powers of $\left|u_{n b}\right|$ (e.g. a quadratic dependence on the near-bed velocity) would lead to an even sharper slope of the growth rate curve near zero.

$\eta$ : this is the coefficient of the nonlinear coupling term. It usually takes values of order one and changes sign depending on $S$ (figure $8 d$ ). The term $\eta \mathscr{A} \mathscr{B}$ tells one by how much the growth rate of sand waves changes if they are superimposed on a large-scale bed distortion $(\mathscr{B})$. A positive (negative) sign of $\eta$ suggests that sand waves on the top of a sand bank will have a larger (smaller) growth rate than sand waves with a zero mean. The sign of $\eta$ depends on the viscosity parameterization. We will examine two viscosity models.

If the simpler model, $v_{t}=$ const, is employed, the value of $\eta$ is always positive. To see this, let us fix some $1 / \lambda$ slightly greater than $1 / \lambda_{c}$ and solve the linear problem with this value of $1 / \lambda$ in two cases: (i) the unperturbed depth is $H$, and (ii) the unperturbed depth is smaller than $H$. The resulting growth rates can be compared, and it turns out that for the smaller depth, the growth rate is always larger. This is easy to understand. When water becomes shallower, the currents must become stronger, and therefore the critical value of $1 / \lambda$ decreases. Thus for a fixed $1 / \lambda$ and shallower water, the value $1 / \lambda-1 / \lambda_{c}$ is larger and the growth rate is larger too.

Next, let us turn to a viscosity model of type (2.1) with $\alpha_{1}<0$. The situation is now more complicated. If the depth becomes smaller, two things happen: (i) the currents have a tendency to become stronger, just as in the previous case, (ii) the near-bed currents are weakened because the viscosity changes. This can be understood in the following way: on the top of a sand wave crest, the depth $(H)$ becomes smaller, but also the viscosity (and therefore, the turbulent boundary layer thickness, $\delta$ ) decreases. Therefore, the effective depth (the ratio $H / \delta$ ) can either decrease or increase depending on where we are in the parameter space. This means that the resulting growth rate can increase or decrease respectively. Thus the coefficient $\eta$ can be either positive or negative.

$\xi$ : this coefficient is responsible for the generation of sand banks as a result of the sand-wave amplitude gradients. It tells us that whenever there is a non-trivial curvature in the sand-wave amplitude, the driving term, $\xi|\mathscr{A}|_{x x}^{2}$, becomes non-zero 

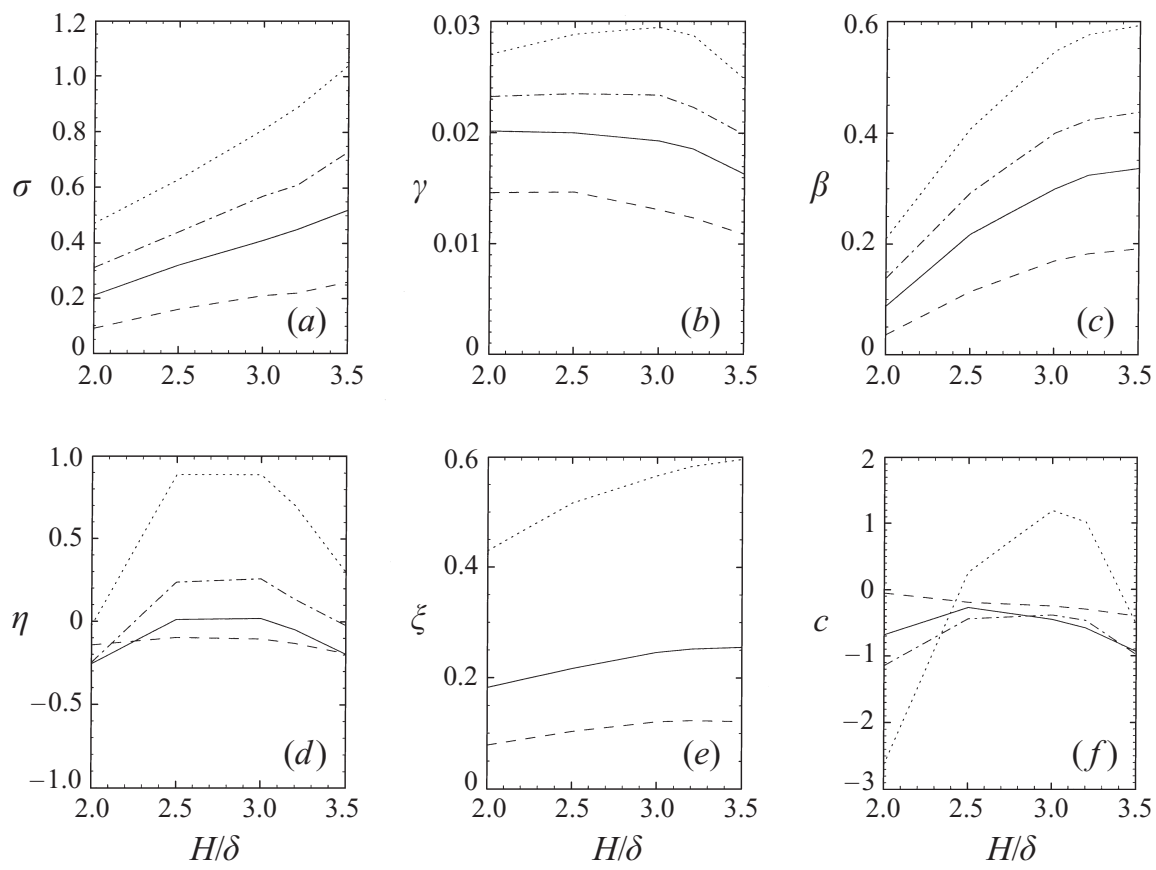

Figure 8. Calculated values for coefficients. Dotted line corresponds to $S=15$, dash-dot to $S=12$, solid line to $S=10$, dashed line to $S=7$.

and forces $\mathscr{B}$ to grow even if it was identically zero initially. This coefficient was found to be non-trivial for all the values of $H / \delta$ and $S$ we have experimented with. For the parameter range under investigation it is always positive and is of the same order as the dispersion coefficient, $\beta$. This is an important result: the nonlinear driving term for sand banks is big enough to compete with the linear damping. The coefficient $\xi$ grows with $S$ and $H / \delta$ (figure $8 e$ ). It appears as a result of the interaction of modes $\Psi_{c}$ and $\Psi_{2(1)}$ as well as the mode $\Psi_{3(0)}^{|A|_{X}^{2}}$ (see the Appendix). The fact that the term $|\mathscr{A}|_{x x}^{2}$ is present in the $\mathscr{B}$-equation can be explained as follows. If the sand-wave amplitude changes with $x$, on a larger scale (the scale of the tidal waves) it looks as if the roughness of the bottom changes with the horizontal coordinate. Therefore, the interaction between the tide and the bottom is different for the patches of large $\mathscr{A}$ and patches of small $\mathscr{A}$. This results in local changes of the bed level, which take place on exactly the same $x$-scale as the changes in $\mathscr{A}$ occur. This fact is reflected in the generating term $\xi|\mathscr{A}|_{x x}^{2}$ in the equation for sand banks.

It is possible to explain why the coefficient $\xi$ is positive. Let us assume that the amplitude of sand waves, $\mathscr{A}$, changes in $x$ over distances much larger than the wavelength of the sand waves. Then the bed looks like a wave on which a slow modulation is imposed (see figure 9). The time-independent flow response consists of (i) vortices created by the sand waves (of horizontal size $2 \pi / k_{c}$ ) and (ii) larger (in horizontal dimension) vortices which are the flow reaction to the slow deformation of the sand-wave amplitude. The competition between the near-bed residual flow corresponding to long bed waves (flow (ii)) on one hand, and gravity on the other hand results in the scale deformations of the mean bed level. The direction of the net sediment flux is then determined by the behaviour of the growth rate curve (figure 3 ) near $k=0$. In our model, $\Gamma(k)<0$ for small $k$, which corresponds to the sediment 


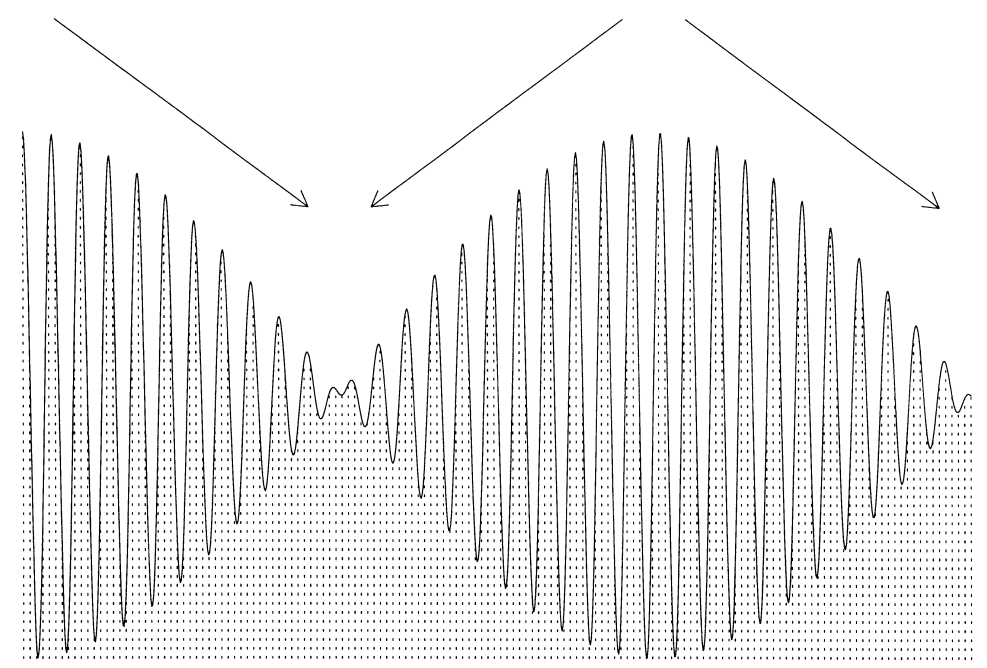

FIGURE 9. The direction of the net sediment flux when the sand wave amplitude is modulated.

flux in the direction from maxima to minima of the function $|A|^{2}$ (see figure 9). Since the mean bed level must be zero, the local depth increases in the spots where the sand waves are the largest. This proves that the generation of $\mathscr{B}$ takes place in such a way that the smallest sand waves are associated with elevations in the sand bed.

$c$ : this is the coefficient responsible for nonlinear saturation of sand wave growth. It was found that its sign depends on position in the physical parameter space. The $(S, H / \delta)$-plane is separated into two regions, where $c$ is negative and positive respectively (figure $8 f$ ). The latter situation only occurs for larger values of $S$.

If there are no sand banks, the nonlinear saturation can only occur because of the term $c|\mathscr{A}|^{2} \mathscr{A}$. However, the mean level distortion contributes into the equation for the sand-wave dynamics. Let us set $\mathscr{B}_{T_{m}}=0$ (a steady state) and estimate the value of $\mathscr{B}$ from the right-hand side of equation (4.22), i.e. $\mathscr{B}=-\left.\xi|\beta| \mathscr{A}\right|^{2}$. Then, this expression can be substituted into equation (4.21) to give a contribution to the term $|\mathscr{A}|^{2} \mathscr{A}$. Let us call the new coefficient in front of this term $\tilde{c}$, so that

$$
\tilde{c}=c-\eta \xi / \beta .
$$

It is the coefficient $\tilde{c}$ which is important for the nonlinear saturation of the sand-wave growth. It was found that there is a large portion of the parameter space where the coefficient $\tilde{c}$ is negative. This means that sand waves are saturated at the third order by means of a cubic nonlinearity. However, in the domains where $\tilde{c}$ is positive, saturation does not occur. In this case, one might try and go up to the fifth order, because the nonlinear saturation could be realized by the term $|\mathscr{A}|^{4} \mathscr{A}$. However, the saturating amplitudes may be so large as to call the whole weakly nonlinear analysis into question.

We have discussed all the terms present in the equations for $\mathscr{A}$ and $\mathscr{B}$ up to the fourth order. Terms such as $\mathscr{A}_{x},|\mathscr{A}|_{x}^{2}$ etc. are not possible because they break the symmetry of the problem (they have a preferred direction, i.e. change sign when $x \rightarrow-x)$.

All the data in figure 8 correspond to sand waves whose wavelengths range from about $800 \mathrm{~m}$ to $1200 \mathrm{~m}$. Regular sand features of this size have been observed on certain North American coast lines (J. Restrepo 1998, personal communication). 
However, wavelengths observed in the North sea fall between approximately $200 \mathrm{~m}$ and $800 \mathrm{~m}$ (Huntley et al. 1993). For this range of wavelengths, we can calculate all the coefficients $(\sigma, \gamma, \eta, \beta, \xi)$ except for the coefficient $c$. The reason for this is the following. The higher the wavenumber is, the harder it is to solve the Navier-Stokes equations numerically. We did not have any problems with the code for a large interval of values of $k_{c}$ (which corresponded to wavelengths from about $700 \mathrm{~m}$ and higher). However, for shorter waves, a problem was encountered when we needed to solve for modes with $k=2 k_{c}$, which was a necessary step when calculating the coefficient $c$. In order to calculate the coefficient $c$ for shorter sand waves, the present numerical scheme would have to be upgraded. All the other coefficients, including $\xi$ and $\beta$ which are most important for our analysis, can be calculated for all wavelengths. Here we present the resulting coefficients for the case of shorter sand waves (corresponding to $H / \delta=4.2$ and $S=12$ ). The critical wavelength turns out to be $500 \mathrm{~m}$ which is a typical value for the North Sea. We have

$$
\sigma=1.3, \quad \gamma=6 \times 10^{-3}, \quad \eta=-0.76, \quad \beta=0.29, \quad \xi=0.41 .
$$

One can see that the coefficients have the same order of magnitude as in figure 8 . We do not expect any qualitative changes in the nonlinear behaviour for sand waves with smaller wavelength. Most importantly, the size of coefficients $\beta$ and $\xi$ and their ratio for sand waves of all sizes have similar values to what we see in figure 8 .

\subsection{Sensitivity to the choice of viscosity model}

The numerical results presented in the previous subsection were obtained for viscosity parameterization I. We have also performed the nonlinear analysis for Model II with $\alpha_{1}<0, \alpha_{2}=0$. It turns out that the numerical values of the coefficients only slightly differ from the ones presented in figure 8 . To illustrate this, let us consider the set of parameters $H / \delta=3.5$ and $S=15$. It turns out that the values $\alpha_{1}=-13, \alpha_{2}=0$ in expression (2.1) give the same critical wavelength as predicted by Model I, namely $801 \mathrm{~m}$. The coefficients in the amplitude equations for the two models compare as follows:

$\begin{array}{lllllll}\text { Model } & \lambda_{c} & \sigma & \gamma & \eta & \beta & \xi \\ \text { I } & 176.0 & 1.0 & 0.025 & 0.30 & 0.48 & 0.60 \\ \text { II } & 188.1 & 1.38 & 0.025 & 0.69 & 1.22 & 1.42\end{array}$

Another example we present here corresponds to parameters $H / \delta=2.5$ and $S=10$. Again, the values $\alpha_{1}=-8, \alpha_{2}=0$ for Model II were chosen to give the same wavelength as in Model I. We obtained the following coefficients:

$\begin{array}{lllllll}\text { Model } & \lambda_{c} & \sigma & \gamma & \eta & \beta & \xi \\ \text { I } & 114.6 & 0.32 & 0.020 & 0.01 & 0.22 & 0.22 \\ \text { II } & 124.9 & 0.34 & 0.020 & 0.30 & 0.34 & 0.38\end{array}$

One can see that the coefficients have the same sign and order of magnitude. A general trend is that the values of $\beta$ and $\xi$ of the equation for sand-bank amplitude are slightly larger for Model II. This is a consequence of the fact that the growth rate curve for this model is steeper near $k=0$ than it is for Model I. The ratio $\xi / \beta$ for Model II is about $5-10 \%$ larger. The conclusion is that the nonlinear excitation mechanism is generic and does not change much when different versions of model (2.1) are used. 

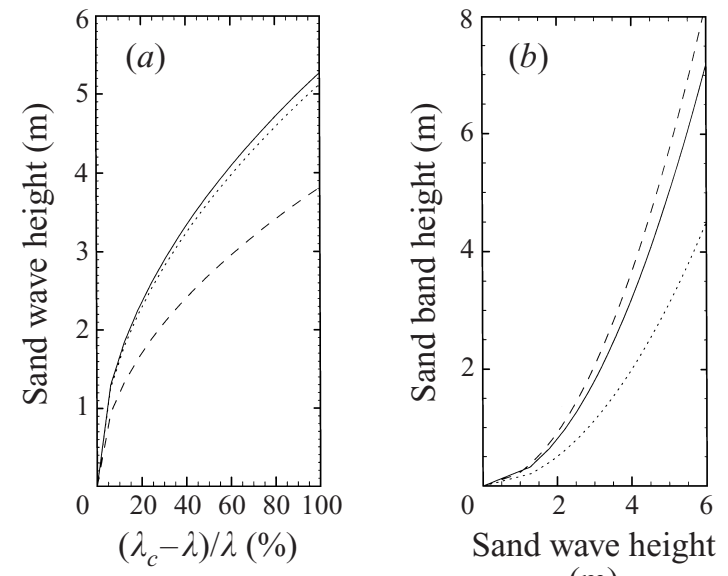

(m)

FIGURE 10. Estimated sand wave and sand bank height. Solid line corresponds to $H / \delta=3.5$ and $S=15$, dotted line to $H / \delta=2.0$ and $S=10$ and dashed line to $H / \delta=2.0$ and $S=12$.

\subsection{Predictions}

Let us write down a typical system (4.21)-(4.22) using explicit numerical values of the coefficients. For $H / \delta=3.5$ and $S=15$, the critical wavelength of the sand waves is $801 \mathrm{~m}$ and $\lambda_{c}=175.9$. This means that as long as the control parameter $\lambda$ is less than $\lambda_{c}$, sand waves are expected to grow. The nonlinear evolution is then described by the equations

$$
\begin{aligned}
\mathscr{A}_{T_{m}} & =1.0\left(\left(\lambda_{c}-\lambda\right) / \lambda_{c}\right) \mathscr{A}+0.025 \mathscr{A}_{\tilde{x} \tilde{x}}-0.52|\mathscr{A}|^{2} \mathscr{A}+0.30 \mathscr{A} \mathscr{B}, \\
\mathscr{B}_{T_{m}} & =0.48 \mathscr{B}_{\tilde{x} \tilde{x}}+0.60|\mathscr{A}|_{\tilde{x} \tilde{x}}^{2} .
\end{aligned}
$$

We can make some estimates. The solution $\mathscr{B}$ can be found from the balance on the right-hand side of equation (5.5), i.e. $\mathscr{B} \approx-1.25|\mathscr{A}|^{2}$. Then, we plug this expression into equation (5.4) which yields $\tilde{c} \approx-0.9<0$. Next, the amplitude of sand waves can be determined from the balance of terms on the right-hand side of equation (5.4) and is $\mathscr{A} \approx \sqrt{\left(\lambda-\lambda_{c}\right) /\left(0.9 \lambda_{c}\right)}$. The dimensional height of sand waves (sand banks) is given by $2 l \mathscr{A}(l \mathscr{B})$, where $l=2.5 \mathrm{~m}$. For example, in order for sand waves to grow up to $1 \mathrm{~m}$, the difference between $\lambda$ and $\lambda_{c}$ must be about 6 , which is about $3 \%$ of the magnitude of $\lambda_{c}$. If $\lambda$ is less than its critical value by about $14 \%$, then the sand waves will grow up to $2 \mathrm{~m}$ etc. The corresponding sand-bank height can be also calculated. The results are summarized in figure 10, where we used the following rough estimates: $\mathscr{A}=\sqrt{\sigma\left(\lambda_{c}-\lambda\right) /\left(\lambda_{c} \tilde{c}\right)}$ for sand-wave height and $\mathscr{B}=\xi \mathscr{A}^{2} / \beta$ for sand-bank height. These estimates reproduce correctly typical sizes of sand patterns observed in nature (see e.g. Rubin \& McCulloch 1980; Wilkens 1997; and figure 12 below).

What is significant is that even though $\lambda-\lambda_{c}$ might be of the same order of magnitude as $\lambda_{c}$ itself (which means that $\epsilon$ is not small), expansion (4.2) still holds. In the previous section, in order to make the procedure of the nonlinear analysis as clear as possible, we considered different orders of $\epsilon$. However, rigorously speaking, the expansion itself was made not in $\epsilon$, but rather in $A \epsilon$. This means that for the expansion to be valid, it is not $\epsilon$, but $\epsilon A$ which must be a small number. For instance, for waves of $5.4 \mathrm{~m}$ height, $\epsilon A$ is still only about 0.2 and $\epsilon^{2} B$ is small as well.

We can make some predictions concerning the dynamics of sand waves and sand banks. It is very interesting that the growth rate of sand banks turns out to be of the 
(b) Linear growth of sand waves

(c) Non-uniform growth

(d) Generation of sand banks

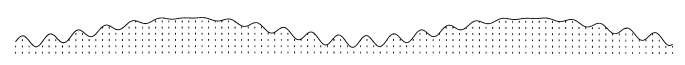

FIGURE 11. Predicted long-scale bed dynamics.

same order of magnitude as the growth rate of sand waves. To see that, let us first calculate the typical time for the growth of sand waves, $\Delta T_{s w}$, using equation (5.4). If $\left(\lambda-\lambda_{c}\right) / \lambda_{c}=0.25$, then the growth rate of sand waves, $\Gamma_{s w}=\sigma\left(\lambda-\lambda_{c}\right) / \lambda_{c}=0.25$, and using $\Delta T=2$ years, we obtain for sand waves that $\Delta T_{s w}=\Delta T / \Gamma_{s w}=7.7$ years which coincides with most field observations. We proceed by using equation (5.5) to find the typical time for the sand-bank generation, $\Delta T_{s b}$. The driving term, $|\mathscr{A}|_{\tilde{x} \tilde{x}}^{2}$, can be estimated to give a rough idea about the growth of sand banks. Let us assume the sand-wave amplitude to be $5 \mathrm{~m}$, and the size of the sand bank $7 \mathrm{~km}$. Then it is easy to show that it takes about $\Delta T_{s b}=10$ years for a sand-bank height to increase by $1 \mathrm{~m}$. This time interval is longer if the sand-wave height is smaller and the horizontal dimension of the sand bank is larger.

In Hulscher (1996), it was shown that a typical time for the linear growth of sand banks was of the order of 200 years. The linear mechanism responsible for their generation was found to rely on horizontal currents rather than on vertical ones (it was shown by Hulscher et al. (1993) that the linear generation of sand banks can be described by a vertically averaged two-dimensional model). The present work does not include this mechanism simply because it does not take into account the second horizontal dimension. In the model we consider, sand banks are strongly damped in the linear problem. However, as follows from the nonlinear analysis, the generation through equation (5.5) takes place on a faster time scale than the linear generation of Hulscher (1996).

The predicted long-scale behaviour of the bed is illustrated in figure 11. If the currents are strong enough, a flat bed is unstable, and sand waves are linearly generated (figure $11 a, b$ ). If their amplitude varies in space (that could for instance happen if the properties of sand are slightly different in different patches of the bed), then the mean level of the bed changes (figure $11 c, d$ ). The signs of coefficients in equations (4.21)-(4.22) are such that the mean level decreases where the local sandwave amplitude is larger and increases where sand waves are smaller. As a result, smaller sand waves are placed on the tops of the generated sand banks, and larger ones are in the valleys. This coincides with observations. In figure 1, sand waves are seen in the troughs of sand banks (note that sand banks are rotated with respect to the tide; this is a result of the Coriolis force and can be captured by a three-dimensional model). Also, the result of figure 11(d) is in agreement with observations of Wilkens (1997), who analysed some existing field data and found (see figure 12) that the height 


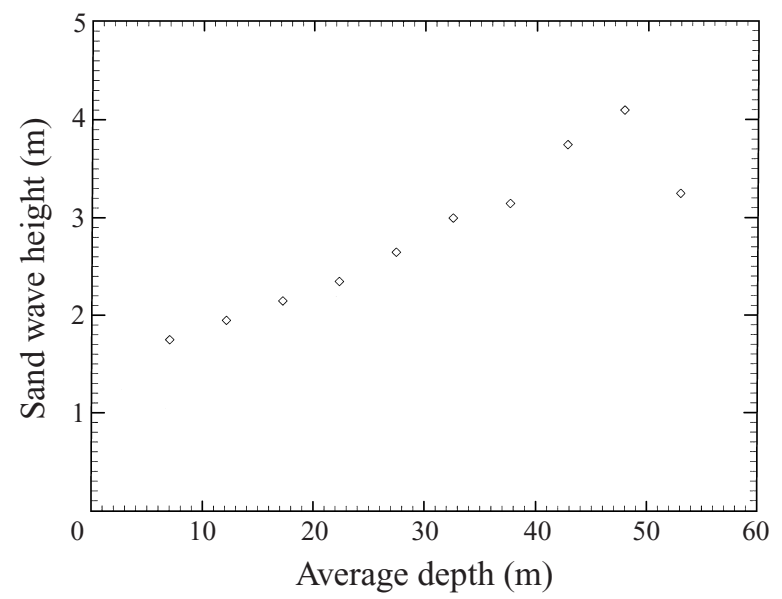

FIGURE 12. Sand wave height distribution over depth, after Wilkens (1997).

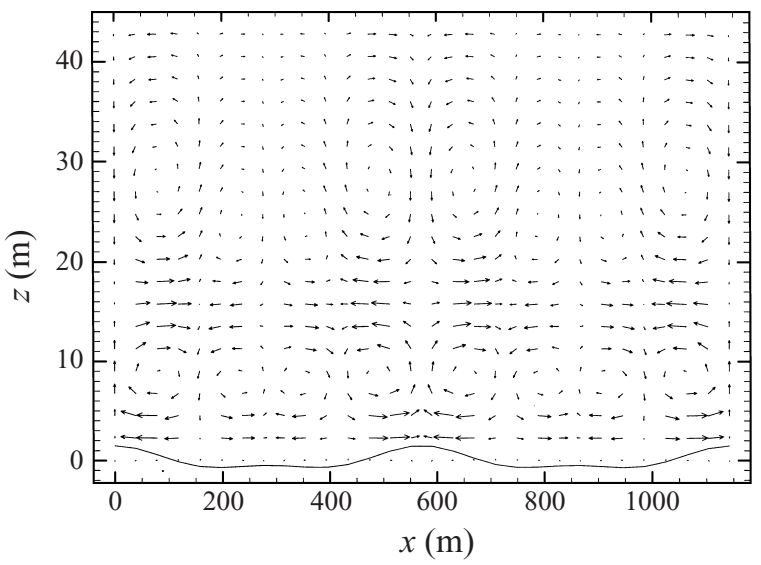

Figure 13. Nonlinear residual flow and bed shape.

of the sand waves grows with the local depth. This indicates that the present model leads to a qualitatively correct nonlinear behaviour of the system.

\subsection{Nonlinear flow response and bed shape}

The water flow adjusts to the sand-wave bed distortion. The response consists of modes $A \Psi_{1} \mathrm{e}^{\mathrm{i} k_{c} x}, A^{2} \Psi_{2(2)} \mathrm{e}^{2 \mathrm{i} k_{c} x}, A_{X} \Psi_{2(1)} \mathrm{e}^{\mathrm{i} k_{c} x}, A B \Psi_{3(1)}^{A B} \mathrm{e}^{\mathrm{i} k_{c} x},|A|^{2} A \Psi_{3(1)}^{|A|^{2} A} \mathrm{e}^{\mathrm{i} k_{c} x}$ etc., see equation (4.2). All these functions contain time-independent harmonics, and therefore contribute to the initial linear cells corresponding to $k=k_{c}$. An example of such nonlinear cells is shown in figure 13. The nonlinear shape of the bed (equation (4.3)) now also contains higher harmonics and is (locally) similar to the shape predicted by Vittori \& Blondeaux (1990) for sand ripples (see figure 13). The main difference is that in our analysis, both the mean level $(\mathscr{B})$ and the sand-wave amplitude $(\mathscr{A})$ are allowed to vary slowly in space.

Let us next consider the flow reaction to the long-wave $\mathscr{B}$-component of the bottom elevation. If $B$ is $X$-independent, then the flow correction will look like $B \Psi_{\text {mean }}$ and have no time-independent component. This is clear from symmetry reasoning. If a time-independent component had an infinite wavelength (i.e. was proportional to 
$\left.\mathrm{e}^{\mathrm{i} k_{c}}\right)$, then the flow would have a unidirectional component, which is not allowed by the symmetries of the problem. Therefore, the flow reaction changes its direction with the tide. Now, if $B$ changes slowly in space, then a time-independent component must appear, because it was proven by the linear analysis that the flow reaction to a non-uniform perturbation contains a residual flow. This manifests itself in the mode $B_{X} \Psi_{3(0)}^{B_{X}}$ (see the Appendix), which only has a time-independent component and makes up for the mode $B \Psi_{\text {mean }}$ not containing it. This residual component has a magnitude about 10 times larger than the magnitude of the initial residual current, $A \Psi_{1}$. This is a direct consequence of the viscosity parameterization. As a result, the coefficient $\beta$ in the amplitude equations is positive and large in comparison with $\gamma$.

Similar behaviour is observed when a long-wave modulation is superimposed on some shorter waves. If $A$ were constant in $X$, the only large-scale nonlinear flow response would be the mode $|A|^{2} \Psi_{2(0)}$. This flow has no time-independent components for symmetry reasons. Now, if $A$ is allowed to vary slowly in space, then the flow will react by producing the harmonic $|A|_{X}^{2} \Psi_{3(0)}^{|A|_{X}^{2}}$ which contains a time-independent component. Again, it is directed downhill with respect to the gradients of $|A|^{2}$ and is rather strong. This results in the fact that the coefficient $\xi$ is positive and of the same order as $\beta$.

\subsection{Further remarks}

Here we will mention some general properties of system (4.21)-(4.22). First, we note that in order to satisfy the requirement that the total amount of sand cannot change, we need to complete the system (4.21)-(4.22) with the condition

$$
\overline{\mathscr{B}}=0 \text {, }
$$

where the bar denotes averaging in space. From equation (5.6) one can immediately see that if $|\mathscr{A}|_{\tilde{x} \tilde{x}}^{2}=0$, then $\mathscr{B}$ satisfies a diffusion equation. If we are interested in stationary solutions, there are two possibilities: $(\mathscr{A}, \mathscr{B})=(0, C \tilde{x})$, where $C$ is some constant, and $(\mathscr{A}, \mathscr{B})=(\mathscr{A}, 0)$. In the latter case, the stationary solution for the envelope $\mathscr{A}$ is $\mathscr{A}=G \mathrm{e}^{\mathrm{i} K \tilde{x}}$ with constants $G$ and $K$ satisfying $\sigma\left(\lambda_{c}-\lambda\right) / \lambda_{c}-\gamma K^{2}+c G^{2}=0$. This corresponds to sand waves with a constant amplitude and the wavenumber in the neighbourhood of $k_{c}$. One can perturb this solution by adding $\rho \mathrm{e}^{\mathrm{i} k l+\mathrm{i} f t}+$ c.c. to the amplitude $G$ and $\mathrm{i} \theta \mathrm{e}^{\mathrm{i} k l+\mathrm{i} f t}+$ c.c. to the phase, $\mathrm{i} K \tilde{x}$. Linear stability analysis shows that there are regimes where such a solution is stable for a finite bandwidth of wavenumbers $K$. The necessary condition for the existence of stable solutions of the form $\mathscr{A}=G \mathrm{e}^{\mathrm{i} K \tilde{x}}, \mathscr{B}=0$ is simply $\tilde{c} \leqslant 0$. If this condition is satisfied, the stability criterion is $(K / G)^{2} \leqslant|\tilde{c}| /(2 \gamma)$ (this is analogous to the Eckhaus boundary).

Next, we consider the case when $|\mathscr{A}|^{2}$ varies in space. $\mathscr{B}$ cannot stay trivial anymore but is forced to change in time and space to satisfy equation (4.22). A simple nontrivial stationary solution of system (4.21)-(4.22) that satisfies condition (5.6) is

$$
(\mathscr{A}, \mathscr{B}), \quad \mathscr{B}=-(\xi / \beta)\left(|\mathscr{A}|^{2}-|\overline{\mathscr{A}}|^{2}\right) .
$$

Note that solution (5.7) can be realized as a stationary state beginning from a $\mathscr{B}=0$ initial condition, because the gradient of $|\mathscr{A}|^{2}$ serves as a driving force for $\mathscr{B}$. Plugging this expression for $\mathscr{B}$ into equation (2.5), we obtain a modified stationary equation for $\mathscr{A}$ :

$$
0=\left[\sigma\left(\lambda_{s}-\lambda\right) / \lambda_{c}-\mu|\bar{A}|^{2}+\gamma \partial_{\tilde{x} \tilde{x}}+\tilde{c}|A|^{2}\right] A,
$$

where $\mu=\eta \xi / \beta$. As one can see, this expression resembles the right-hand side of the 
well-known Ginsburg-Landau equation (GLE) with real coefficients. Solutions of the GLE relevant to patterns can be found in Cross \& Hohenberg (1993).

Now we will concentrate on the situation when the values of $\mathscr{A}$ and $\mathscr{B}$ are close to the solution $(A, B)=(0, C \tilde{x})$ as the initial condition. This solution represents a uniform slope with no sand waves and is unstable to perturbations of $\mathscr{A}$. Even a very small deviation of $\mathscr{A}$ from zero will lead to a very large positive contribution to the growth rate (through the last term of equation (4.21)), if $|\tilde{x}|$ is large enough and $\tilde{x}$ has the right sign. For $\eta>0(\eta<0)$, sand waves appear in the shallow (deep) part of the bed. On the other hand, for $\mathscr{B}$ smaller (larger) than some constant, all perturbations of $\mathscr{A}$ will decay. This will lead to the following situation: where the water is deep (shallow), the bottom is flat. Towards shallower (deeper) waters, there will be some sand waves, their amplitudes growing towards the shallower (deeper) ocean. Of course, changes in $\mathscr{A}$ will contribute in equation (4.22) and change the slope, $\mathscr{B}$. An approximate stationary solution for $\mathscr{A}$ corresponding to $B=C \tilde{x}$ (let us assume $C>0$ ) can be found, namely

$$
A= \pm \sqrt{a_{0}+a_{1} \tilde{x}}
$$

where $a_{0}=-\sigma\left(\lambda_{c}-\lambda\right) /\left(\lambda_{c} c\right)$ and $a_{1}=-\eta C / c$ (this can be obtained by substituting expression (5.9) into equation (4.21) and ignoring the diffusion term). Note that solution (5.9) becomes exact in the limit $|\tilde{x}| \rightarrow \infty$. It is likely that for the situation of a uniform slope as the initial condition, the resulting solution for $\mathscr{A}$ will look like expression (5.9) in the shallow (deep) part of the sea for $\eta>0(\eta<0)$, and $\mathscr{A}=0$ for large negative (positive) values of $\tilde{x}$. Some interesting front dynamics can be expected where the two parts of the solution 'meet'.

In general, the stationary solution for $\mathscr{B}$ can be written as $\mathscr{B}=-\xi / \beta\left(|\mathscr{A}|^{2}-|\overline{\mathscr{A}}|^{2}\right)+$ $C \tilde{x}$. Then $\mathscr{A}$ satisfies equation

$$
\left[\sigma\left(\lambda_{c}-\lambda\right) /\left(\lambda_{c} c\right)-\mu|\overline{\mathscr{A}}|^{2}+\gamma \partial_{\tilde{x}}^{2}+\tilde{c}|\mathscr{A}|^{2}+\eta C \tilde{x}\right] \mathscr{A}=0,
$$

which can be transformed to the Painleve equation of the second kind. Appropriate solutions of this equation and their connection formulae can be found, for instance, in Fokas \& Its (1993) and Flaschka \& Newell (1980). We will not discuss them here.

\subsection{Wavelength doubling}

Finally, we would like to mention an interesting scenario which takes place when $1 / \lambda$ is strongly supercritical. If one looks at the growth-rate dependence on $k$ for different values of $\lambda$, it is easy to notice that the larger the difference $\left|1 / \lambda-1 / \lambda_{c}\right|$, the wider the band of excited modes centring at $k=k_{c}$. As $1 / \lambda$ increases, there will be a point $\lambda=\lambda_{1 / 2}\left(\lambda=\lambda_{2}\right)$ when the mode with $k=k_{c} / 2\left(k=2 k_{c}\right)$ becomes excited. Let us for instance consider the former case. The subharmonic mode with $k=k_{c} / 2$ can nonlinearly (quadratically) couple with the most excited $\left(k=k_{c}\right)$ mode. Let us denote the amplitude of the mode with $k=k_{c} / 2$ as $\mathscr{A}_{1 / 2}$. Then the coupling of modes $\mathscr{A}$ and $\mathscr{A}_{1 / 2}$ can be illustrated by the following system of equations:

$$
\begin{gathered}
\mathscr{A}_{t}=\sigma\left(\left(\lambda_{c}-\lambda\right) / \lambda_{c}\right) \mathscr{A}+c_{1} \mathscr{A}_{1 / 2}^{2}, \\
\left(\mathscr{A}_{1 / 2}\right)_{t}=\sigma_{1 / 2}\left(\left(\lambda_{1 / 2}-\lambda\right) / \lambda_{1 / 2}\right) \mathscr{A}_{1 / 2}+c_{2} \mathscr{A}_{1 / 2}^{*},
\end{gathered}
$$

where the star means the complex conjugate and $\sigma_{1 / 2}=\lambda_{1 / 2} \partial \Gamma / \partial(-\lambda)$ estimated at $\lambda=\lambda_{1 / 2}$. Here we omitted all gradients and higher-order nonlinearities. Note that such nonlinear coupling is only possible because the time dependence of the sandwave modes contains no travelling waves, i.e. the critical mode does not depend on 
the fast time (obviously, $\Gamma_{c}=0$ ). If it did, we would require the frequency of $k_{c}$ to be twice that associated with $k_{c} / 2$ (or close to it). From equation (5.12) one can see that as long as $\sigma_{1 / 2}\left(\lambda_{1 / 2}-\lambda\right) / \lambda_{1 / 2}+c_{2} \operatorname{Re} \mathscr{A}>0$, the mode with $k=k_{c} / 2$ will grow. Since $\mathscr{A}$ is a complex function, condition $\lambda<\lambda_{1 / 2}$ is sufficient for the mode $\mathscr{A}_{1 / 2}$ to grow, because $\operatorname{Re} \mathscr{A}$ can be always adjusted by choosing the appropriate phase of the complex envelope $\mathscr{A}$. Moreover, $\mathscr{A}_{1 / 2}$ can even grow subcritically (if it is not too strongly linearly damped).

This kind of an instability can be seen from the geometry of the flow. Let us assume that modes with $k=k_{c}$ and $k=k_{c} / 2$ are superimposed. First we make a further assumption that the relative phase shift of the two modes is zero, i.e. the resulting picture is such that every other crest of sand waves is slightly smaller. Then, the residual flow will consist of two superimposed sequences of vortices (with periods of $2 \pi / k_{c}$ and $\left.4 \pi / k_{c}\right)$. The flow response generated by the bed mode $\mathscr{A}_{1 / 2} \mathrm{e}^{\mathrm{i} k_{c} / 2 x}$ will enhance the flow of those initial vortices which are directed towards the crests of the bigger bumps, and weaken the initial vortices adjacent to the smaller bumps. Therefore, the bigger bumps will grow and the smaller ones will be slowly washed out. In the end, the mode with $k=k_{c} / 2$ will prevail and a wavelength doubling will take place. In a more general situation, when initially modes with $k=k_{c}$ and $k=k_{c} / 2$ are superimposed and shifted in phase, the resulting flow will act in such a way that the sand waves with amplitude $\mathscr{A}$ will gradually move and eventually their crests will coincide with the crests of the mode $\mathscr{A}_{1 / 2}$. This will bring us to the situation described before. The drifting of the initial sand wave towards the crests of mode $k=k_{c} / 2$ is exactly what was meant by the complex mode $\mathscr{A}$ choosing the appropriate phase to enhance the growth of the mode $\mathscr{A}_{1 / 2}$.

Finally, for the mode with $k=2 k_{c}$, the equations will look exactly like equations (5.11)-(5.12), except that the role of the mode $\mathscr{A}_{1 / 2}$ will be played by mode $\mathscr{A}$, and the mode $\mathscr{A}$ will be replaced by the mode with $k=2 k_{c}$. As a result of the nonlinear interaction, the mode $\mathscr{A}$ will win over the mode with $k=2 k_{c}$ (in other words, the shortening of the wavelength does not occur).

\section{Conclusions}

In this paper we have found a system of two partial differential envelope equations that couples the amplitude of sand waves and the mean bed level dynamics. This system predicts the generation of sand banks where the amplitude of sand waves changes in space. The nonlinear mechanism found here is rather generic. For instance, it has been shown that it does not depend on the particular form of the eddy viscosity parameterization, as long as $\left\langle v_{t}\right\rangle$ is smaller over crests of the bed distortion than it is in troughs. We have also demonstrated that the nonlinear mechanism of sand-bank formation is as strong or stronger than the linear mechanism described in Hulscher (1996).

The typical horizontal scale of sand banks is defined by the characteristic distance on which sand waves change. This in turn can depend on several things, e.g. (i) how supercritical the system is (in other words, how large the band width of excited modes is), and (ii) some external reasons. The latter means that parameters such as the tidal strength, the sand roughness, the local depth etc., are not constants. In real seas they vary from place to place. Consequently, the control parameter, $\lambda$, can be different in different patches of the bed, and the sand-wave behaviour (for instance, the growth rate) can have a horizontal structure. According to equations (4.21)-(4.22), this will lead to the generation of sand banks. 
The methods used for the nonlinear analysis presented in this paper can easily be applied to other physical situations. For instance, one can perform a similar analysis for a system describing sand bed dynamics under sea waves ebbing and flowing on a shore line. It was predicted by Blondeaux (1990) that the linear instability leads to the generation of periodic features whose wavelength is similar to the observed wavelength of sand ripples. The symmetries of the latter problem are exactly the same as in the present problem. One difference is the ratio $H / \delta$, which is much larger in the case of ripples than it is in the case of sand waves. Another difference is the appropriate viscosity model (it is an eddy viscosity parameterization in our case and the molecular water viscosity in the case of ripples). Despite these two differences, the nonlinear procedure is analogous to the one described in this paper. The resulting equations are expected to look similar to equations (5.4)-(5.5) except that $\mathscr{A}$ will describe the amplitude of sand ripples and $\mathscr{B}$ the megaripples, which can be treated as a varying mean bed level. We expect that the term $\eta \mathscr{A} \mathscr{B}$ will disappear in case of sand ripples. Indeed, since the depth in this problem can be though to be infinite, it is unlikely that the mean depth level changes can influence the growth rate of sand ripples. This will be a generalization of the results of Vittori \& Blondeaux (1990), where the nonlinear analysis was performed for sand ripple dynamics, but neither spatial gradients of the sand ripple envelope nor the mean flow mode were taken into account.

One problem with the analysis described in this paper is that it requires very lengthy calculations. All the terms which appear in the resulting envelope equations can be found just by looking at symmetries of the problem, so the most time-consuming part is calculating the numerical values of the coefficients in these equations. In order to do that one has to (numerically) solve a number of systems of inhomogeneous ordinary differential equations with boundary conditions. The flow solution obtained is then used to find all the terms in the sediment equation. The result is that the predictions based on the envelope equations are qualitatively reasonable and estimates have the correct order of magnitude.

It is characteristic for this problem that even though at each step one has to solve a full hydrodynamical system, only a small part of the flow information is then used for the sand evolution equation. Namely, one only needs derivatives of the stream function evaluated at the bed (and not as functions of $z$ ). This suggests that there might be an alternative way to tackle the problem of modelling the behaviour of a sandy bed in tidal seas. Namely, one could try and avoid solving the Navier-Stokes equation and, instead, write down an empirical (Swift-Hohenberg-like) equation for the time evolution of the bed level, $h$. In other words, it might be possible to replace the hydrodynamical system coupled with the sediment conservation law, equations (2.5)(2.8), with one equation describing the time behaviour of the bottom profile. All the hydrodynamical processes can be effectively included in the parameters (coefficients) of this phenomenological equation. Such a model would be expected to grasp all the important features of the bed dynamics (the linear instability of sand waves as well as the long-term behaviour of the system and the excitation of a long bed wave due to the sand-wave intensity curvatures). The coefficients in this equation could be adjusted to match the observations. They would contain the necessary information about the water flow over the sandy profile, which in the present model is obtained by means of solving the full hydrodynamical system.

The two ways (the analysis presented in this paper on one hand, and writing down an empirical equation, on the other) correspond to the microscopic and macroscopic approaches in mathematical modelling. In the case of sand patterns, the microscopic 
way has been studied in this paper in great detail. The other way still needs to be explored.

\section{Appendix}

Here we will use ideas presented in $\S 4$ and perform a weakly nonlinear analysis of a tidal solution over a flat bed. We will use the eddy viscosity model (2.2). The calculations for model (2.1) are very similar.

\section{A.1. Order $\epsilon^{2}$}

At the second order, we get the following results:

(i) For $m=0$, the solvability condition is satisfied automatically $\left(\mathscr{G}_{2(0)} \equiv 0\right)$. The first term in solution (4.13) is specifically included in expansion (4.2) with $h_{2(0)} \equiv B$. The second term turns out to be driven by nonlinear terms proportional to $|A|^{2}$, which defines the dependence of this mode on the slow variables. For $m=0$, there are only three boundary conditions for the differential equation, which are (4.5), (4.7) and (4.10), so that $\Psi_{2(0)}^{n l}$ is defined up to a constant.

Now let us show that condition (4.10) is satisfied by a solution of a system of the same type as (4.4)-(4.7) if the limit $k \rightarrow 0$ is taken in the end. This is very similar to what happens in system (3.6)-(3.11) with $k=0$. We write down the following expression:

$$
\Psi=\Psi_{0}+\epsilon A \mathrm{e}^{\mathrm{i}\left(k_{c}+\kappa / 2\right) x} \Psi_{1}\left(k_{c}+\kappa / 2\right)+\epsilon A^{*} \mathrm{e}^{-\mathrm{i}\left(k_{c}-\kappa / 2\right) x} \Psi_{1}^{*}\left(k_{c}-\kappa / 2\right),
$$

where function $\Psi_{1}$ is the solution of the linear problem (3.6). In the limit $\kappa \rightarrow 0$, expression (A 1) becomes exactly the expansion (4.2) up to first order in $\epsilon$. The mode (20) appears as a result of a nonlinear interaction of the mode $\Psi_{1}$ with itself (more precisely, the interaction of the component $A E_{c}$ with its complex conjugate). Expansion (A 1) allows for a small phase mismatch $(\kappa)$ between the two modes, which will be taken to zero later on. Let us substitute expansion (A 1) in our system. The resulting equation can be written as

$$
\mathscr{L}_{\kappa} \tilde{\Psi}_{2(\kappa)}=F_{n l}(\kappa),
$$

where the right-hand side comes from nonlinear interaction between the modes in expansion (A 1). It is easy to prove that the solution of this system, which we denoted as $\tilde{\Psi}_{2(\kappa)}$, in the limit $\kappa \rightarrow 0$ satisfies system (4.4)-(4.9) for the (20) mode. It is possible to check that the normalization condition (given by equation (4.10) for the mode (20)) follows from the boundary conditions of the system for $\Psi_{2(\kappa)}$ in the limit $\kappa \rightarrow 0$. Therefore, we conclude that an alternative way of obtaining mode $\Psi_{2(0)}$ is given by

$$
\Psi_{2(0)}=\lim _{\kappa \rightarrow 0} \Psi_{2(\kappa)} .
$$

If we did not have to obtain mode $\Psi_{3(0)}$, we would not have to go into these details. However, we will need mode (30) for our solvability conditions, and we will use system (A 2) to calculate it.

(ii) For $m=1$, it is more convenient to use the function $\tilde{\Psi}$ defined in equation (3.16). It turns out that the system for $\tilde{\Psi}_{2(1)}^{n l}$ can be written as

$$
\mathscr{L}_{\left(k=k_{c}\right)} \tilde{\Psi}_{2(1)}^{n l}=-\left.\mathrm{i} A_{X}\left(-\frac{\partial \mathscr{L}}{\partial k} \tilde{\Psi}_{c}+\frac{\partial \mathscr{F}}{\partial k}\right)\right|_{k=k_{c}},
$$

where $A_{X} \equiv \partial A / \partial X, \mathscr{F}$ is the same as in (3.16) and the boundary conditions are 
homogeneous (note that in this case the superscript $n l$ is slightly misleading, this mode is only driven by the slow variation of $A$ in $X$ rather than by nonlinear interactions). On the other hand, if we differentiate equation (3.17) with respect to $k$ at $k=k_{c}$, we get

$$
\frac{\partial \mathscr{L}}{\partial k} \tilde{\Psi}_{c}=-\mathscr{L} \frac{\partial \tilde{\Psi}_{1}}{\partial k}+\left.\frac{\partial \mathscr{F}}{\partial k}\right|_{k=k_{c}} .
$$

Therefore, we can take

$$
\Psi_{2(1)}^{n l}=-\left.\mathrm{i} A_{X} \frac{\partial \Psi_{1}}{\partial k}\right|_{k=k_{c}} .
$$

Next, we notice that because of equation (A 6), the left-hand side of solvability condition (4.14) can be written as

$$
A_{T_{1}}-\left.\mathrm{i}\left(A_{X} \frac{\partial \mathscr{D}}{\partial k}+A_{X} \frac{\partial \Gamma}{\partial k}\right)\right|_{k=k_{c}}=0
$$

where $\mathscr{D}$ is the the left-hand side of growth rate relation (3.14) and is therefore equal to zero. The $k$-derivative of the growth rate at the critical conditions is also equal to zero. Thus we have the solvability condition at the second order,

$$
A_{T_{1}}=0 \text {. }
$$

Note that the general expression for the (21) mode is,

$$
\Psi_{2(1)}=h_{2(1)} E_{c} \Psi_{c}-\left.\mathrm{i} A_{X} E_{c} \frac{\partial}{\partial k} \Psi_{1}\right|_{k=k_{c}}
$$

The reason why the mode with amplitude $A_{X}$ enters the expansion is that the amplitude $A$ is space dependent so that $\partial_{x}$ is not exactly equal to $\mathrm{i} k_{c}$. That is why the right-hand side of all equations can be represented as a $k$-derivative of the right-hand side at the previous order. The amplitude $h_{2(1)}$ can be taken to be zero without loss of generality.

(iii) For $m=2$, the amplitude $h_{2(2)}$ turns out to be proportional to $A^{2}$. Therefore function $\Psi_{2(2)}$ in equation (4.2) is also proportional to $A^{2}$.

\section{A.2. Order $\epsilon^{3}$}

Let us continue writing out terms in expansion (4.2). At the third order of $\epsilon$ we have

$$
\begin{aligned}
\epsilon^{3}\left(\frac{1}{2} \Psi_{3(0)}(X, T, z, t)+\right. & E_{c} \Psi_{3(1)}(X, T, z, t) \\
& \left.+E_{c}^{2} \Psi_{3(2)}(X, T, z, t)+E_{c}^{3} \Psi_{3(3)}(X, T, z, t)+\text { c.c. }\right) .
\end{aligned}
$$

In our analysis, we will only need modes (30) and (31) (the former enters solvability condition (4.12) and the latter solvability condition (4.14)).

(i) For $m=0$, we note that the slow behaviour of mode $\Psi_{3(0)}$ comes from two contributions, $B_{X}$ and $|A|_{X}^{2}$. This can be seen from the fact that the terms driving the mode are proportional to either $B_{X}$ or $|A|_{X}^{2}$. In order to make the analysis more clear, we will separate the mode (30) according to this, by writing

$$
\Psi_{3(0)}=\Psi_{3(0)}^{B_{X}}+\Psi_{3(0)}^{|A|_{X}^{2}} .
$$

Next, we note that the equation for mode $\Psi_{3(0)}^{B_{X}}$ can be written as

$$
\mathscr{L}_{(k=0)} \tilde{\Psi}_{3(0)}^{B_{X}}=-\left.\mathrm{i} B_{X}\left(-\frac{\partial \mathscr{L}}{\partial k} \tilde{\Psi}_{\text {mean }}+\frac{\partial \mathscr{F}}{\partial k}\right)\right|_{k=0},
$$


where we used the function with tilde defined in equation (3.16) in order to make the boundary conditions homogeneous. One can see the resemblance of equation (A 12) to equation (A 4). As before, we can set

$$
\Psi_{3(0)}^{B_{X}}=-\left.\mathrm{i} B_{X} \frac{\partial \Psi_{1}}{\partial k}\right|_{k=0} .
$$

Exactly as the mode with $A_{X}$, the mode $\phi_{3(0)}^{B_{X}}$ arises to reflect the fact that there are slow variations in $x$, and even for $(n 0)$ modes, $\partial_{x}$ is not exactly 0 . The technique we use to calculate the components of this mode is to 'pretend' that there is a small $x$-dependence in the phase (instead of $B \mathrm{e}^{0 \mathrm{i} k_{c} x}$ we consider $\left.B \mathrm{e}^{\mathrm{i}\left(0 k_{c}+\kappa\right) x}\right)$ and then take a $\kappa$-derivative at the point $\kappa=0$.

We shall use a similar idea for calculating the mode $\phi_{3(0)}^{|A|_{X}^{2}}$. It appears owing to nonlinear interactions between modes $A E_{c}$ and $A^{*} E_{c}^{-1}$ (by $A^{*}$ we denote the complex conjugate of $A$ ), whose amplitude is allowed to slowly vary is space. Again, even though the sum of the phases $\left(E_{c} E_{c}^{-1}\right)$ is zero, the slow modulation in $x$ must be taken into account. Therefore, by analogy, we can think of the equation for the mode $\phi_{3(0)}^{|A|_{X}^{2}}$ as a $k$-derivative of another equation, namely, the equation for the mode (20).

Of course, the equation for mode (20) does not contain $k$ because it corresponds to a zero power of $E_{c}$. Instead, we can use equation (A2). It was obtained using expansion (A 1). The small mismatch, $\kappa$, between the two interacting modes models the fact that there is a non-trivial $x$-derivative of the product $A E_{c} A^{*} E_{c}^{-1}$. Now let us consider the structure of the driving term for the mode $\phi_{3(0)}^{|A|_{X}^{2}}$. In the general case of complex $A$, the time-independent component turns out to be proportional to $A_{X} A^{*}+A_{X}^{*} A=|A|_{X}^{2}$, whereas the time-dependent component is proportional to $\left(A_{X} A^{*}-A_{X}^{*} A\right)$. If $A$ is real, the time-dependent part of this mode vanishes. Note that in the solvability condition, we will only need the time-independent component (the rest gets averaged out to zero). Now, if $A$ is real, it is easy to see that that equation for the mode $\Psi_{3(0)}^{|A|_{X}^{2}}$ can be written as

$$
\mathscr{L}_{(k=0)} \tilde{\Psi}_{3(0)}^{|A|_{X}^{2}}=-\left.\mathrm{i}|A|_{X}^{2}\left(-\frac{\partial \mathscr{L}_{\kappa}}{\partial \kappa} \tilde{\Psi}_{2(\kappa)}+\frac{\partial F_{n l}(\kappa)}{\partial \kappa}\right)\right|_{\kappa=0},
$$

where function $F_{n l}(\kappa)$ is the same as in equation (A 2). Using the same argument as in the case of modes with $A_{X}$ and $B_{X}$, we simply set

$$
\Psi_{3(0)}^{|A|_{X}^{2}}=-\left.\mathrm{i}|A|_{X}^{2} \frac{\partial \Psi_{2(\kappa)}}{\partial \kappa}\right|_{\kappa=0} .
$$

This calculation was checked by using a direct method of finding the mode $\Psi_{3(0)}^{|A|_{X}^{2}}$ (in the case of a general complex $A$ ). The time-independent part is of course the same in the two methods. Except, the method described here is more convenient. It contains fewer steps (i.e. the differential equations only have to be solved twice, in first and second orders of $\epsilon$ ), whereas the direct method also requires the solution of the system in the third order. This means that the time-truncation must be done three times instead of two. Every time one has to truncate, the accuracy decreases by about $5-15 \%$. Therefore, the procedure described in this section is preferable.

Note that the function $\Psi_{3(0)}$ that we found here corresponds to the second term in expression (4.13), whereas the first contribution was taken to be zero from the start. Mode $\Psi_{3(0)}$ does not enter the solvability condition until the next order in $\epsilon$. At third 
order in $\epsilon$, equation (4.12) simply gives

$$
\mathscr{G}_{3(0)}=-B_{T_{1}}=0 .
$$

(ii) $m=1$. Mode $\Psi_{3(1)}$ from expansion (A 10) can be found in a straightforward way from the corresponding equations. It is driven by terms proportional to $A B$ and $A|A|^{2}$ (again, we take $h_{3(1)}=0$ ). Using this mode, the results for the second-order modes and equation (4.14), we obtain the following solvability condition:

$$
A_{T_{2}}=\sigma / \lambda_{c} A+\gamma \partial_{X}^{2} A+c|A|^{2} A+\eta A B,
$$

where $\sigma, \gamma, c$ and $\eta$ are real constants.

\section{A.3. $O r d e r \epsilon^{4}$}

In order to find a non-trivial generator of the $B$-term we have to go to the fourth order in $\epsilon$. Using the expressions for the third-order mode $\Psi_{3(0)}$ and the $m=0$ solvability condition, we obtain

$$
B_{T_{2}}=\beta \partial_{X}^{2} B+\xi \partial_{X}^{2}|A|^{2},
$$

where $\beta$ and $\xi$ are real constants. There is no contribution into the $A$-dynamics in this order $\left(A_{T_{3}}=0\right)$.

Remark. As already mentioned in connection with mode $\Psi_{3(0)}^{|A|_{X}^{2}}$, all the modes related to slow derivatives (namely the modes $\Psi_{2(1)}, \Psi_{3(0)}^{B_{X}}$ and $\Psi_{3(0)}^{|A|_{X}^{2}}$ ) can be obtained in a direct way by writing out the corresponding driving force (which comes from $A_{X}$, $B_{X}$ and $|A|_{X}^{2}$ respectively) and solving the resulting equations. However, the way presented in this analysis gives a better intuition about why these modes appear. Also, numerically this method is more precise. The alternative way was used for double checking the results.

\section{REFERENCES}

Bagnold, R. A. 1956 The flow of cohesionless grains in fluids. Proc. R. Soc. Lond. A 249, 235-279. BAILARD, J. A. 1981 An energetics total load sediment transport model for a plane sloping beach. J. Geophys. Res. 86 (C11), 10938-10954.

Bailard, J. A. \& InMan, D. L. 1981 An energetics bedload model for a plane sloping beach: local transport. J. Geophys. Res. 86 (C3), 2035-2043.

Blondeaux, P. 1990 Sand ripples under sea waves. Part 1. Ripple formation. J. Fluid Mech. 218, $1-17$.

Bowden, K. F. 1983 Physical Oceanography of Coastal Waters. Ellis Horwood.

Bryan, K. 1969 A numerical method for the study of the circulation of the world ocean. J. Comput. Phys. 4, 347.

Cross, M. C. \& Hohenberg, P. C. 1993 Pattern formation outside of equilibrium. Rev. Mod. Phys. 65, 851-1112.

De Swart, H. E. \& Zimmerman, J. T. F. 1993 Rectification of the wind-driven ocean circulation on the beta plane. Geophys. Astrophys. Fluid Dyn. 71, 17-41.

De VRIEND, H. J. 1990 Morphological processes in shallow tidal seas. In Residual Currents and Long-term Transport (ed. R. T. Cheng), pp. 276-301, Springer.

Dyer, K. R. 1986 Coastal and Estuarine Sediment Dynamics. John Wiley.

Dyer, K. R. \& HuntLey, D. A. 1999 The origin, classification and modelling of sand banks. Cont. Shelf Res. 19, 1285-1330.

Dyer, K. R. \& Soulsby, R. L. 1988 Sand transport the continental shelf. Ann. Rev. Fluid Mech. 20, 295-324. 
Engelund, F. 1970 Instability of erodible beds. J. Fluid Mech. 42, 225-244.

Falques, A., Montoto, A. \& Iranzo, V. 1996 Bed-flow instability of the longshore current. Cont. Shelf Res. 16, 1927.

Flaschka, H. \& Newell, A. C. 1980 Monodramy and spectrum preserving deformations. Commun. Math. Phys. 76, 65-116.

FoKAS, A. S. \& ITS, A. R. 1993 The isomonodromy method and the Painlevé equations. In Important Developments in Soliton Theory (ed. A. S. Fokas \& V. E. Zakharov), pp. 99-122. Springer.

Fredsoe, J. 1974 On the development of dunes in erodible channels. J. Fluid Mech. 64, 1-16.

Fredsoe, J. \& ENGELUnd, F. 1975 Bed configurations in open and closed alluvial channels. Institute of Hydrodynamics and Hydraulic Engineering, Technical University of Denmark, Series Paper 8.

Gerkema, T. 1998 A note on the effect of finite Stokes-layer thickness in a morphodynamic stability problem. In Physics of Estuaries and Coastal Seas (ed. J. Dronkers \& M. B. A. M. Scheffers), pp. 387-395. Balkema.

Gerkema, T. 1999 A linear stability analysis for tidally generated sand waves. J. Fluid Mech. submitted.

Hulscher, S. J. M. H. 1996 Tidal-induced large-scale regular bed form patterns in a threedimensional shallow water model. J. Geophys. Res. 101 (C9), 20727-20744.

Hulscher, S. J. M. H., De Swart, H. E. \& De Vriend, H. J. 1993 Generation of offshore tidal sand banks and sand waves. Cont. Shelf Res. 13, 1183-1204.

Huntley, D. A., Huthnance, J. M., Collins, M. B., Liu, C.-L., Nicholls, R. J. \& Hewitson, C. 1993 Hydrodynamics and sediment dynamics of North Sea sand waves and sand banks. Phil. Trans. R. Soc. Lond. A 343, 461-474.

KNAAPEN, M. A. F. 1999 On the modeling of rhythmic morphological patterns using a GinzburgLandau equation. Civil Engineering \& Management Rep. 99W-007/MICS-004. University of Twente, The Netherlands.

Komarova, N. L. \& Hulscher, S. J. M. H. 2000 Linear instability mechanisms for sand wave formation. J. Fluid Mech. 413, 219-246.

Komarova, N. L. \& Newell, A. C. 1995 The mean flow driven by sandbar instabilities. In Nonlinear Dynamics and Pattern Formation in the Natural Environment (ed. A. Doelman \& A. van Harten), pp. 147-167. Longman.

LARCOMBE, P. \& JAGO, C. F. 1996 The morphological dynamics of intertidal megaripples in the Mawddach Estuary, North Wales, and the implications for palaeoflow reconstruction. Sedimentology 43, 541-559.

Leeder, M. R. 1982 Sedimentology: Process and Product. G. Allen and Unwin.

MAAS, L. R. M. \& HAREN, J. J. M. van 1987 Observations on the vertical structure of tidal and internal currents in the central North Sea. J. Mar. Res. 45, 293-318.

Newell, A. C. \& Moloney, J. V. 1992 Nonlinear Optics. Addison-Wesley.

Newell, A. C., Passot, T. \& Lega, J. 1993 Order parameter equations for patterns. Ann. Rev. Fluid Mech. 25, 399.

PARKER, G. 1976 On the cause and characteristic scales of meandering and braiding in rivers. J. Fluid Mech. 76, 457-480.

Pedlosky, J. 1987 Geophysical Fluid Dynamics, 2nd edn. Springer.

Phillips, O. M. 1977 The Dynamics of the Upper Ocean, 2nd edn. Cambridge University Press.

Pinardi, N., Rosati, A. \& PACANOWSKi, R. C. 1995 The sea surface pressure formulation of rigid lid models. Implications for altimetric data assimilation studies. J. Mar. Syst. 6, 109-119.

Prandtl, L. 1932 Zur turbulenten Stroeming in Roehren und langs Plaetten. Ergebn. Aerodyn. Versuchsanst, Goettingen 4, 18-29 (in German).

Rubin, D. M. \& McCulloch, D. S. 1980 Single and superimposed bedforms: a synthesis of San Francisco Bay and flume observations. Sedimentary Geol. 26, 207-231.

Schielen, R., Doelman, A. \& De Swart H. E. 1993 On the nonlinear dynamics of free bars in straight channels. J. Fluid Mech. 252, 325-356.

SchuttelaARs, H. M. \& De Swart, H. E. 1996 An idealized long-term model of a tidal inlet. Eur. J. Mech B/Fluids 15, 55-80.

Seminara, G. \& Tubino, M. 1992 Weakly nonlinear theory of regular meanders. J. Fluid. Mech. 244, 257-288.

SoulsBy, R. L. 1990 Tidal current boundary layers. In The Sea, vol. 9, part A (ed. B. le Mehaute, \& D. M. Hanes), pp. 523-566. John Wiley and Sons. 
Soulsby, R. L. 1997 Dynamics of Marine Sands: A Manual for Practical Applications. London: Telford.

Stride, A. H. (Ed.) 1982 Offshore Tidal Sands: Processes and Deposits. Chapman and Hall.

Tennekes, H. \& Lumley, J. L. 1972 A First Course in Turbulence. MIT Press.

VAN RiJn, L. C. 1993 Handbook of Sediment Transport by Currents and Waves. Aqua Publ., Amsterdam.

Vittori, G. \& Blondeaux, P. 1990 Sand ripples under sea waves. Part 2. Finite-amplitude development. J. Fluid Mech. 218, 19-39.

Wilkens, J. 1997 Sandwaves and possibly related characteristics. Report for Alkyon Hydraulic Consultancy and Research. 